\title{
PROFESSOR ROBERT COURRIER
}

\section{AN INTERVIEW}

\section{7th and 18th May 1972}

Ruth Deanesly. Il est vraiment très aimable à vous d'avoir accepté de nous expliquer votre vie et votre œuvre. Cela sera d'un grand intérêt pour les endocrinologistes de la reproduction en Angleterre et dans maintes parties du monde.

Vous pourriez peut-être commencer en nous parlant un peu de vos premières années et de vos services militaires en 1914-19?

Robert Courrier. Je suis né à Saxon-Sion, le 6 octobre 1895 d'un ménage d'instituteurs. Saxon est un petit village de Lorraine au pied de Sion, la 'Colline inspirée', lieu de pélerinage chanté par Maurice Barrès. Mon père, d'origine vosgienne, était un grand travailleur, il était très bien noté et fut assez rapidement nommé Directeur d'une Ecole à Pont-à-Mousson où j'ai fait mes études secondaires.

J'ai passé le premier baccalauréat en 1912, dans la section Latin-Sciences. En 1913 ce fut le baccalauréat de Mathématiques élémentaires et celui de Philosophie. Puis je me suis inscrit, en novembre 1913, à la faculté des Sciences de Nancy pour obtenir le certificat de Physique, Chimie et Sciences naturelles qui permettait d'entrer dans une faculté de Médecine. Je voulais être médecin militaire de manière à ne plus être, assez tôt, à la charge de mes parents. A Nancy, j'ai connu mon ami Jacques Benoit, qui suivait aussi les cours de la faculté des Sciences. Nous avons passé nos examens en juillet 1914, et ce fut la guerre. Au mois d'août je fus infirmier volontaire dans le collège de Pont-àMousson qui était à quelques kilomètres de la frontière et qu'on avait transformé en hôpital auxiliaire. Une nuit, j'étais de garde auprès d'un officier allemand blessé quand les allemands bombardèrent notre collège. Sur son toit on avait peint une grande croix rouge. J'ai dit à l'officier allemand qui parlait très bien le français "Voilà vos amis qui bombardent la Croix Rouge". Il m'a répondu: "Moi, je ne suis pas artilleur".

Au mois de novembre 1914, j'entrais à la faculté de Médecine de Nancy, j'y ai pris ma première inscription. Chaque matin, nous allions à l'hôpital pour panser les blessés qui étaient fort nombreux. A la fin du mois de décembre, je fus mobilisé. Avant mon départ pour l'armée, on m'a donné une deuxième inscription de Médecine. J'ai été incorporé dans le $19^{\circ}$ bataillon de Chasseurs-àpied. Je suis parti pour le front en avril 1915. Mon bataillon se battait dans les forêts d'Argonne où se livraient des combats acharnés car le Kronprinz voulait absolument percer le front français. Nous avions beaucoup de pertes et on manquait d'infirmiers au bataillon. Comme j'étais le seul étudiant en Médecine, on m'a nommé infirmier.

D. En anglais nous disons 'medical orderly'.

C. Comme infirmier, j'ai participé, naturellement, à de nombreux combats. Tout d'abord en Argonne en mai 1915; ensuite, dans un endroit que l'on appelle les "Eparges", village au pied d'une colline, non loin de Verdun, là se livrèrent 
des combats sanglants au mois de juin pour occuper la colline. En septembre 1915, c'est la grande attaque française de Champagne; nous sommes revenus 370 sur 1000 hommes du bataillon. Nous restons dans le même secteur plusieurs mois et faisons la connaissance des gaz asphyxiants allemands. En juin 1916, mon bataillon est envoyé à Verdun. La bataille de Verdun est célèbre dans l'histoire, c'était un carnage, et c'est au cours de cette bataille que j'ai reçu la Croix-de-guerre. En octobre 1916, autre bataille, cette fois dans la Somme. G'est là qu'un éclat d'obus tombe sur la tranchée; heureusement il ricoche sur le parapet puis vient me frapper derrière l'oreille. L'éclat était gros, mais il avait perdu sa force vive et je n'ai été simplement qu'assommé. Mais je restais fatigué, j'avais de la fièvre et finalement au bout de deux ou trois jours on m'a évacué. J'ai été en traitement à l'hôpital d'Amiens, puis en convalescence.

A cette époque, on manquait de médecins auxiliaires. Tous les étudiants en médecine qui étaient au front depuis deux ans environ furent appelés au Val-de-Grâce, le grand hôpital militaire de Paris. Pendant quelques mois, on nous a fait suivre des cours pour nous apprendre un peu le métier de médecin, de médecin du front bien entendu. Après ces quelques mois, on nous a nommés médecins auxiliaires et j'ai été désigné pour le front de Salonique, à l'Armée d'Orient, avec un beau croissant sur mon képi. Nous arrivons trente-cinq médecins auxiliaires à Salonique, c'était à l'automne de 1917. Trois jours après mon arrivée, gros accès de fièvre. On m'envoie à l'hôpital où l'on me dit: "vous avez la dingue". La 'dingue méditerranéenne' est due à la piqûre d'un moustique appelé, je crois, le phlébotome; c'est beaucoup moins grave que le paludisme. Je suis resté à l'hôpital environ trois semaines. Mais au cours de ces trois semaines, les trente-quatre médecins auxiliaires qui étaient venus avec moi avaient été répartis dans les diverses unités. Quand je suis sorti de l'hôpital, on s'est dit : "que va-t-on faire de celui-là ? on ne va pas le renvoyer en France". Je fus affecté à un camp de prisonniers, à $15 \mathrm{~km}$ de Salonique. C'était 'le camp de Mikra'. Il y avait là quelques Turcs et beaucoup de Bulgares. Le service était assuré par un docteur en Médecine; j'étais le médecin auxiliaire, le médecin en second. Je me rappelle que $\mathrm{j}$ 'avais organisé un poulailler. Le matin j'allais chercher les œufs avant de passer la visite; je donnais des œufs frais aux prisonniers malades, qui étaient enchantés. Mais une épidémie de typhus se déclara dans le camp, l'autre médecin était rentré en France, j'étais alors seul et pas très rassuré. On m'a organisé une installation qu'on appelait la 'station d'épouillage' : douches, autoclaves, etc. ... Quand un malade était atteint de typhus dans une tente, je l'envoyais à l'hôpital de Salonique et j'isolais immédiatement ses camarades; on brûlait tout ce qu'il y avait dans la tente pour détruire les poux.

Au bout d'un certain temps, la guerre s'est terminée au front d'Orient et c'est dans cette région que l'armistice a débuté.

D. Et après l'armistice?

C. Nous recevons une division allemande prisonnière qui se battait en Serbie. A peine arrivé, le médecin chef allemand 'l'Oberstabsarzt', vient me trouver; ' $j$ 'ai des malades, beaucoup de paludisme, je n'ai pas de quinine". Je lui réponds "combien en voulez-vous de kilos ? "Et je lui ouvre l'armoire qui en renfermait des kilos. "Wunderbar!" 
A un certain moment, je reçois un ordre du service de santé de Salonique: "On va vous envoyer des femmes et des enfants qui viennent d'Odessa. Il y a eu des cas de typhus à bord. Comme vous avez une station d'épouillage, vous allez pouvoir les traiter". C'était des femmes dont les maris travaillaient en Russie comme ingénieurs, banquiers, industriels, ils étaient Français, Belges, Suisses, Hollandais. Les maris étaient restés en Russie pour essayer de sauver ce qu'ils pouvaient sauver, mais ils avaient envoyé leurs femmes et leurs enfants à Odessa où on les embarquait. On en a amené à Salonique des milliers dans plusieurs bateaux. C'est un souvenir épouvantable. Ces femmes, nous étions obligés de les traiter comme nous traitions les prisonniers, à la station de douches. C'est-à-dire qu'elles passaient dans une salle de déshabillage. On prenait leurs vêtements pour les mettre à l'autoclave pendant qu'elles se douchaient. C'était très désagréable pour elles. J'ai téléphoné à Salonique réclamant des infirmières et des infirmières sont venues nous aider. Je me rappelle une petite histoire assez drôle. Avec un convoi de femmes, arrive un jour une troupe de tziganes. On les fait entrer dans la salle de déshabillage. On prend tout ce qu'il y avait dans leurs valises pour l'autoclave. Après la douche, on leur rend leurs vêtements. Hélas, ces femmes gagnaient leur vie en dansant avec des robes couvertes de paillettes. A l'autoclave toutes les paillettes avaient fondu. Le chef de la troupe voulait me tuer; pendant tout le temps qu'ils sont restés là, ces pauvres tziganes, je ne pouvais sortir qu'encadré de deux tirailleurs sénégalais.

Fin avril 1919, le médecin général du service de santé de l'armée d'Orient me convoque et me dit "Eh bien, mon cher, le typhus s'est bien terminé, vous êtes passé à travers. Vous n'avez eu ni typhus, ni paludisme".- "Oh ! dis-je, je prenais de la quinine tous les soirs".- "Ni dysenterie amibienne. On a encore besoin de vous pour quelque temps ici, mais choisissez: ou bien vous rentrez en France tout de suite si vous le voulez; ou bien vous restez avec la médaille des épidémies"'. J'ai répondu que je préférais rentrer en France. Il m'a tout de même proposé pour la médaille des épidémies. Je pense que c'est mon successeur qui l'a eue. J'arrivai à Toulon, le $\mathrm{l}^{\text {er }}$ mai 1919.

D. Et pourquoi êtes vous allé à Strasbourg?

C. Mon père venait d'être nommé directeur de l'École de l'Académie à Strasbourg. Je vais donc à Strasbourg. Je suis démobilisé assez vite, et je m'inscris à la faculté de Médecine. J'y retrouve mon ami Jacques Benoit. Je suis en particulier les cours de $\mathbf{M}$. Bouin, qui, de Nancy, avait été nommé par Poincaré à Strasbourg; il faisait un enseignement sur l'ovaire, qui m'intéressait beaucoup. M. Bouin venait aux travaux pratiques et interrogeait les étudiants. Il avait beaucoup de bienveillance pour les vieux militaires que nous étions. Comme je lui demandais des explications sur des parties du cours que je n'avais pas bien comprises, il me dit: "Si cela vous intéresse tellement, venez donc au laboratoire"'. Voilà comment je suis entré au laboratoire de M. Bouin assez tôt, en été 1919.

A. Jost. Mais Monsieur, n'aviez-vous pas connu M. Bouin à Nancy ?

G. Non. J'avais connu son frère qui était professeur de zoologie à la faculté des Sciences.

Après quatre ans et demi de guerre, je n'ai plus songé à faire la médecine militaire. 
D. Naturellement, vous désiriez des études différentes.

C. Tout en poursuivant mes études de médecine et de licence ès sciences, je fréquentais de plus en plus le laboratoire qui m'intéressait énormément. Monsieur Bouin m'avait dit: "Il faut avant tout apprendre la technique histologique. Allez à l'abattoir, vous prélèverez, sur des porcs, oviductes et ovaires. Je vous demande d'étudier les modifications de l'oviducte en rapport avec les transformations de l'ovaire'. C'est ainsi que j'ai commencé, et vous constaterez que cela eut une grande influence sur ma vie. Voici la suite.

Un jour d'hiver, on apporte au laboratoire une chauve-souris vivante. Un anatomiste qui se trouvait là, s'efforce de découvrir ses organes génitaux. Dans le laboratoire de M. Bouin vous savez qu'on étudiait avant tout la physiologie génitale. L'anatomiste ouvre le ventre de cette chauve-souris, il ne trouve rien et jette l'animal. Après son départ, j'examine cette chauve-souris, je n'en avais jamais vu de près. Sûrement c'était un mâle, car chez les chauves-souris, le pénis est indépendant. En disséquant soigneusement, non pas dans le ventre, mais sous la peau de chaque côté du pénis, je trouve de part et d'autre deux formations attachées l'une à l'autre: une petite formation ronde et en dessous, quelque chose de beaucoup plus gros; de ce quelque chose de plus gros se détachait un conduit qui allait derrière la vessie: c'était sans doute le canal déférent. Alors naturellement j'ai fixé tout de suite ces organes et j'ai coupé et coloré. En examinant les coupes avec M. Bouin, nous avons été surpris. La petite formation supérieure était un testicule au repos, avec des grandes et des petites cellules comme dans un testicule embryonnaire; en dessous, la grosse formation était en réalité la queue de l'épididyme qui était bourrée de spermatozoïdes. Cela plaisait beaucoup à $\mathrm{M}$. Bouin évidemment, de voir la lignée séminale au repos, avec une glande interstitielle bien développée et des caractères sexuels actifs. L'épididyme rempli de spermatozoïdes avait un épithélium en activité sécrétoire. En outre les vésicules séminales et la prostate étaient hypertrophiées. M. Bouin me dit: "Il faut faire une note". Ce fut ma première publication (1), après avoir observé quelques autres chauves-souris mâles d'hiver.

J. C'était votre première publication scientifique en janvier 1920, huit mois après votre arrivée à Strasbourg. Je trouve très intéressant de voir que le titre se termine par: 'I'existence d'une sécrétion épididymaire chez la chauvesouris hibernante; sa signification". Ce n'était pas de la description, c'était de la physiologie.

C. De l'histo-physiologie, oui, tout de suite. Naturellement cela tombait exactement dans la sphère d'intérêt de $M$. Bouin. A partir de ce moment-là, j'ai recherché activement des chauves-souris. Les ouvriers qui réparaient la cathédrale de Strasbourg, y trouvaient facilement en hiver des chauves-souris endormies, des pipistrelles et des sérotines. Je leur achetais ces précieux hibernants. J'ai naturellement constaté que tous les mâles se comportaient exactement de la même façon. Chez les femelles d'hiver, j'ai remarqué aussi un autre phénomène extraordinaire: l'utérus gonflé était rempli de sperme. Les spermatozoïdes étaient rangés régulièrement la tête contre l'épithélium utérin qui sécrétait; ils semblaient être au râtelier comme des chevaux à l'écurie. 
J. C'était l'objet de votre deuxième note (2).

C. En examinant l'ovaire, je ne trouvais pas trace de corps jaune, mais un follicule commençait à grossir. Il ne renfermait pas encore de liquide folliculaire, mais il était déjà volumineux, c'était celui qui allait ovuler au réveil printanier. En somme, il s'agissait d'une phase folliculaire débutante, figée par l'hibernation. La lumière du vagin était oblitérée par un bouchon épithélial kératinisé. Chez le mâle, l'accouplement ayant eu lieu, le testicule rentrait au repos. Mais l'hibernation survenait au moment où le tube séminifère était déjà au repos, et où l'interstitielle ne l'était pas. Ce qui produisait une dissociation magnifique entre le tube séminifère, l'interstitielle et les caractères sexuels secondaires qui allaient exactement de pair avec la glande interstitielle.

Il faut se rappeler qu'en ces temps anciens sévissait la grande bataille de l'interstitielle. Quantité de biologistes affirmaient que l'hormone mâle provenait du tube séminifère et non pas de l'interstitielle. Bouin et Ancel soutenaient le contraire. En 1924, une réunion de l'Association des Anatomistes s'était tenue à Strasbourg. Bouin analysait le déterminisme des caractères sexuels secondaires chez le mâle, et Ancel étudiait la phase progestative chez la Lapine. Il y avait là des adversaires de l'interstitielle, Champy en tête. Je vois encore M. Bouin rappelant ses anciens travaux avec Ancel sur le testicule ectopique, et citant les travaux de ses élèves: Aron sur les Batraciens, Benoit sur les Oiseaux, Courrier sur les Mammifères à spermatogénèse périodique et sur les Poissons. Notre Maître termina son exposé en disant: “ $\mathrm{j}$ 'ai l'impression, Messieurs, que des nuées d'orage s'amoncellent au-dessus de ma tête”. Une discussion violente éclata en effet. C'était très excitant pour nous, les jeunes d'alors.

J. M. Bouin a même été accusé d'être malhonnête, le pauvre homme.

C. Mais oui. Discussion effroyable. Champy soutenait que mes observations sur la chauve-souris étaient fausses, pour lui "quand les caractères sexuels secondaires sont actifs, il n'y a pas d'interstitielle; il n'y en a jamais eu chez les Poissons". Demandant la parole, je m'écriai: "à entendre M. Champy, je suis absolument persuadé qu'il n'a jamais vu un testicule de chauve-souris d'hiver". Derrière moi, se trouvait M. Julin, le "père" Julin comme nous l'appelions (c'était un vieil histologiste belge); il me dit: "jeune homme, si vous étiez mon élève, je vous tirerais les oreilles". Dans les couloirs, après la séance, cela bouillonnait; Champy déclara: "Bouin et Ancel ont fait venir des étudiants pour faire la claque en haut de l'amphithéâtre!" et Géry d'ajouter: "la claque, elle devrait être sur votre figure". Géry, élève de Pierre Masson, était chargé du cours d'Anatomie pathologique. Vous pouvez juger de l'ambiance.

D. Pourquoi Champy était-il si furieux ?

C. Champy a raconté des histoires absolument fausses. Il a déclaré par exemple: "chez le Hérisson en pleine activité génitale, il n'y a pas d'interstitielle". En réalité, elle est énorme chez le Hérisson en activité. Champy craignait qu'à la retraite de M. Prenant, M. Bouin ne demande la Chaire de Paris. Mais M. Bouin n'y tenait pas du tout. Le pauvre M. Prenant se lamentait: “j'ai deux élèves que j'aime beaucoup, Bouin et Champy, et les voilà qui vont se battre!"

Ma troisième note en 1921 a été sur la Thyroïde (3). 
This work, partly inspired by the experiments of Gudernatsch on tadpoles, concerned the effects of feeding thyroid to cats and immature rats. Dustin of Brussels had reported that thyroid feeding had caused thymus regression, but Courrier found that this was a starvation effect and could be prevented if the animals received a rich diet. Fresh pig thyroids from the slaughterhouse were fed to cats kept at home, which caused domestic difficulties! At autopsy, thyroids from these cats were seen to be grey, not the normal pink; sections showed large vesicles full of colloid, with a flattened epithelium. After a study of other thyroid experiments in the literature, Courrier concluded that thyroid feeding had caused the cats' own thyroids to become hypo-functional, a significant discovery, reviewed in later works $(16,40,43)$. Thyroid feeding did not affect testis function in rats (55).

C. A Bruxelles M. Dustin (il fut Recteur de l'Université de Bruxelles), avait constaté que lorsqu'on donnait à manger de la thyroïde à des animaux, le thymus involuait. Il estimait que le thymus était une réserve de nucléo-protéines, et que la thyroïde sécrétait une hormone spéciale qui provoquait la libération des nucléines de cette réserve. La thyroïde m'intéressait aussi parce que Gudernatsch avait réalisé, avant la première guerre, une expérience très spectaculaire et devenue célèbre. Il avait donné des petits fragments de thyroïde fraîche à des têtards de Batraciens, et il avait déclenché une métamorphose brusquée. Devant ces deux observations, je réfléchissais: "Ce serait bien intéressant de donner de la thyroïde à des animaux; j'analyserais la théorie de Dustin et, en même temps, $j$ 'essaierais de provoquer une spermatogenèse précoce chez des impubères, puisque l'on obtient une métamorphose précoce chez les Batraciens". Ma première note étudie l'action de la thyroïde sur le thymus. J'ai constaté qui si le thymus fondait sous l'influence de l'alimentation thyroïdienne, c'était tout simplement parce que les animaux maigrissaient, ils étaient en état de jeûne. En donnant avec la thyrö̈de une alimentation riche, non seulement le thymus ne dégénérait pas, mais il s'hypertrophiait. En réalité, il n'y avait certainement pas une hormone particulière de la thyroïde qui commandait le métabolisme nucléinien du thymus. Sans donner de thyroïde, il suffit de soumettre un animal au jeûne pour voir fondre son thymus.

Pour étudier l'action de la thyroïde sur le testicule impubère, $j$ 'allais à l'abattoir et prélevais cette glande sur les porcs en même temps que les ovaires et les oviductes. Les jeunes chats adoraient se nourrir de thyroïde fraîche, ils en absorbaient de grandes quantités. Mais il fallait leur donner en plus une alimentation très riche pour éviter l'amaigrissement et la mort. Comme à cette époque, le laboratoire manquait de technicien, c'était au domicile de mes parents que je conservais mes jeunes chats pour les nourrir à volonté. Ces animaux, soumis au régime thyroïdien, n'étaient pas propres et ma pauvre maman dut changer plusieurs fois de femme de ménage au cours de ces expériences.

C'est ainsi qu'a commencé ma carrière de chercheur sous la direction d'un Maître illustre et telles sont mes trois premières publications dans trois directions qu'en définitive j'ai suivies toute ma vie: études sur le mâle, sur la femelle, sur la thyroïde. 
L'action sur la thyroïde elle-même: je m'en suis occupé aussi par hasard. Je donnais donc de la thyroïde à ingérer à des chats et à des rats impubères pour examiner l'influence sur le testicule et sur le thymus. Or la thyroïde n'est pas éloignée du thymus et j'ai constaté que la thyroïde des animaux soumis à l'alimentation thyroïdienne était très différente d'une thyroïde normale. Elle n'était plus rose, elle était grise. Je l'ai examinée au microscope et lui ai trouvé un aspect extraordinaire. Ce n'était plus du tout la thyroïde d'un petit animal normal; elle était constituée par des vésicules volumineuses, pleines de colloïde, avec un épithélium extrêmement plat. Quand j'ai vu cette thyroïde gonflée de collö̈de,je me suis demandés'il s'agissait de l'action homo-stimulante dont parlaient à l'époque les médecins qui faisaient de l'opothérapie. J'étais perplexe car on ne connaissait pas bien alors l'histophysiologie de la thyroïde. J'ai fait de la bibliographie pour savoir si l'on avait trouvé des cas où, vraiment, la thyroïde était en hypofonctionnement ou en hyper-fonctionnement, et si l'on avait étudié son histologie dans ces conditions.

Si l'on supprime les $4 / 5$ de la thyroïde (je crois que c'est Roussy qui a fait l'expérience), l'animal est tout d'abord hypothyroïdien; mais au bout d'un certain temps, il va beaucoup mieux. Quand on examine le petit reliquat de glande, on constate que les vésicules sont petites, avec très peu de colloïde, mais, leur épithélium est très élevé. C'était donc là un aspect certain d'hyperfonctionnement. D'autre part, j'ai étudié la biologie des têtards. On sait que les têtards qui se développent dans des ruisseaux de montagne dont l'eau est froide, ont souvent une métamorphose retardée. Il s'agit sûrement d'une déficience thyroïdienne car, quand on donne de la thyroïde à ces animaux, leur métamorphose se produit rapidement. J'ai examiné les travaux des auteurs et pour eux: "la thyroïde de ces têtards, qui est sûrement en hypo-fonctionnement, est constituée de larges vésicules remplies de colloïde et leur épithélium est très plat'. Par conséquent j'avais mis la thyroïde des Rats et des Chats au repos, ce n'était pas une action homo-stimulante de l'opothérapie, mais bien une action homo-inhibitrice".

J. Monsieur, est-ce que vous me permettez? J'ai sous les yeux votre tiré à part de 1928, dans une revue (55) que, malheureusement, on ne trouve plus.

C. Ah oui. Je crois que le titre était: "Action de la thyroïde sur le thymus, sur le testicule et sur la thyroïde", n'est-ce pas?

J. Oui, c'est cela. Voici ce que vous écriviez: "tout se passe comme si la thyroïde sécrétait naturellement son hormone en quantité strictement réglée sur les besoins de l'organisme, à ce point que si cette hormone est artificiellement fournie du dehors, la glande entre au repos. Il semble y avoir une relation entre la quantité d'hormone qui circule dans le milieu intérieur et l'activité glandulaire. C'est une sorte d'équilibre glandulo-hormonal'. Je crois qu'il y avait déjà cette conception dans certaines de vos notes antérieures à celle-là.

G. Certainement. Je crois que j'ai commencé à parler de ces résultats en 1922 et en 1924 à la Société de Biologie $(16,40)$.

Ce que je tiens à signaler, c'est que les points de départ de mes recherches sont dûs au hasard. J'étais dans le laboratoire de $M$. Bouin où l'on étudiait la physiologie génitale du mâle et de la femelle. Le hasard a voulu que je tombe sur une chauve-souris et que les chauves-souris mâles et femelles aient un com- 
portement sexuel vraiment singulier. La chauve-souris mâle a été le point de départ de ma thèse de Doctorat ès Sciences sur les animaux à spermatogenèse périodique: j'ai étudié plusieurs espèces de Chauves-souris, la Taupe, le Hérisson et la Marmotte. Ensuite, c'est par le plus grand des hasards que je fus amené à étudier l'action de l'alimentation thyroïdienne sur le corps thyroïde. C'est en examinant le thymus, pour répondre à Dustin, que j'ai vu cette thyroïde à l'aspect particulier, qui était celui du repos fonctionnel.

From 1921 to 1924 during his medical studies, Courrier published notes and papers on a variety of topics, e.g. a new trypanosome found in bats $(6,7,31)$, and the spermatheca of the queen bee (12) in which, as in bats, his interest lay in the nutrition of the stored sperm by secretory granules from the nucleus. At Strasbourg, Aron was studying batrachians and Benoit, birds. M. Bouin said to Courrier "What about fish? Have you time?" and Courrier agreed to work on them and visited Roscoff Marine Biological Station where he studied fertilization in Echinus $(22,24,33,34)$ and secondary sexual characters of various fish including the blenny $(13,21)$, and also male crabs feminized by the parasite, Sacculinus: unexpectedly these were found to contain testes in full spermatogenesis (11). It was concluded, and later confirmed by others, that in Arthropods male secondary sexual characters were not controlled by the testes.

Courrier made a detailed study of the normal and experimental stickleback, Gasterosteus aculeatus; this had fine, seasonal, secondary sexual characters whose development coincided with that of the interstitial tissue which followed completion of spermatogenesis. If winter sticklebacks were put into warm water, they would come into full spermatogenesis as in summer, but neither the interstitial tissue nor the secondary sexual characters were stimulated $(8,10,14,17,18,41)$.

When Courrier began to collect bats, he naturally also became interested in female reproductive physiology and particularly in the growth and secretion of the follicle. He had seen actively secreting epithelium in the oviducts of pig and dog coinciding with enlargement of the follicles in the ovary $(19,44)$. In 1923, he observed that thickening of the vaginal epithelium in the guinea-pig occurred at first oestrus and was therefore not dependent on the presence of the corpus luteum as Stockard and Papanicolaou had stated (23). Like others at that period, Courrier injected fluid from ripe follicles into immature and castrated mammals and also into hibernating hedgehogs with varying results (39). $\mathrm{He}$ also examined the local action of follicular fluid on the mouse vagina (42).

In 1924, Professor Schickele, the gynaecologist, sent him samples obtained at operations; one, later revealed as amniotic fluid, was particularly active as an oestrogen. While experimenting on new-born guinea-pigs, Courrier decided also to inject follicular fluid into a female shortly before parturition; at birth the young had open vaginae. Contrary to the findings of other workers, whose test animals were mice 
or rats, this experiment showed that oestrogens could cross the placenta (38).

J. Vous n'avez pas publié beaucoup d'illustration à ce sujet, je crois. Vous en avez donné dans votre livre sur l'Endocrinologie de la Gestation (193).

C. A un certain moment $\mathrm{j}$ 'hésitais au sujet de ma carrière et $\mathbf{M}$. Bouin me dit: "mon Cher, ou bien vous faites du laboratoire, ou bien vous faites l'internat en médecine. Mais je ne veux pas que vous fassiez l'un et l'autre à la fois. Vous n'avez qu'à choisir'. J'ai choisi le laboratoire et non la clinique et mon ami Fred Weiss, qui devait devenir professeur de Chirurgie à Strasbourg, m'a dit alors, "tu vas passer ta vie à découper des intestins de grenouille en rondelles?".

Mais je posais des questions à $M$. Bouin au sujet de mon avenir, car je n'avais pas de fortune. L'avenir, c'était le concours d'agrégation et l'enseignement car le C.N.R.S. n'existait pas encore. Je ne pouvais pas avoir d'emploi important à Strasbourg, car mes amis Aron et Benoit étaient devant moi. L'agrégation d'histologie et d'embryologie se passait tous les trois ans, or il y avait neuf facultés de Médecine en France, y compris Alger, et il n'y avait pas neuf places vacantes d'agrégés tous les trois ans. L'avenir était incertain.

En attendant que je puisse passer l'agrégation, $M$. Bouin constatant que j'étais vraiment "mordu", que je voulais coûte que coûte faire du laboratoire, put me trouver un petit traitement de préparateur.

J. Quand avez-vous terminé vos études à la faculté de Médecine?

C. J'ai passé ma thèse de Médecine à Strasbourg en 1924 (39) et l'agrégation en 1925 à Paris. J'ai été nommé à Alger en 1926. Pendant les vacances de 1927, je suis revenu à Strasbourg pour passer ma thèse de Sciences (52).

Pourquoi suis-je allé à Alger? Quand on était reçu à l'agrégation à ce moment-là, on était seulement déclaré agrégeable, mais on n'était pas sûr d'avoir un poste. J'avais assisté à un Congrès des Anatomistes à Turin, j'y avais rencontré $M$. Weber qui m'avait dit: 'je suis Professeur d'Histologie et d'Embryologie à la faculté de Médecine d'Alger. Mais, je suis en mission à Genève où je me plais beaucoup. Je ne retournerai pas à Alger. Si vous voulez y aller, on pourrait créer un poste d'agrégé pour vous. "J'en ai aussitôt parlé à $\mathbf{M}$. Bouin et au Doyen Weiss, celui-ci répondit: "Alger? vous vous rendez compte de ce que vous quittez en allant là-bas? La Fondation Rockefeller vient d'offrir un magnifique Institut à votre Maître Bouin. Vous allez quitter Strasbourg pour aller dans un endroit où vous serez surchargé d'enseignement et où l'onne fait pas de recherche". Nous avons beaucoup hésité ma femme et moi; je m'étais marié en 1923 avec une jeune fille qui était professeur de lycée; nous nous étions rencontrés au laboratoire de Roscoff. En fin de compte, mon père, qui était un vieux fonctionnaire, déclara: "Tu veux faire une carrière dans l'Université, dans les facultés de Médecine. A Strasbourg tu n'es qu'assistant, à Alger tu seras agrégé, Chef de service, et tu hésites!"

J. Il faut ajouter que vous étiez un assistant quand même un peu différent de la majorité d'entre eux. A ce moment-là, vous aviez déjà fait beaucoup de recherches, sur l'ovaire, sur la thyroïde, sur le testicule. Vous aviez de quoi vous lancer dans un laboratoire tout seul.

C. J'étais décidé à accepter Alger après ce que m'avait dit mon père, et $\mathbf{M}$. 
Bouin ajouta: "je suis navré, mais peut-être votre père a-t-il raison". Puis le Doyen de la faculté de Médecine d'Alger écrivit à $M$. Bouin: "que ce $\mathbf{M}$. Courrier vienne, on a besoin de lui, on lui donnera un poste d'agrégé, puisque le titulaire est en mission".

Ma femme, qui était Professeur au Lycée de Colmar, demande alors un poste au lycée d'Alger; elle l'obtient tout de suite. Pour moi, j'avais la lettre du Doyen qui m'invitait à venir. Nous arrivons à Alger dans les derniers jours de septembre 1926. Je vais aussitôt saluer le Doyen, il me conduit au laboratoire d'Histologie qui comprenait trois petites pièces, et je lui demande "Monsieur le Doyen, quand vais-je être nommé Agrégé? Je suis agrégeable par le concours, mais il faut que la Faculté de Médecine me propose au Ministère". "Oui, oui, on va s'en occuper". Mais le ton de cette réponse me laissa inquiet. Par la suite j'ai compris pourquoi. Le Chef de Travaux d'histologie était 'agrégeable' comme moi, mais en chirurgie, et il n'était pas sûr d'avoir un poste d'agrégé de chirurgie. Or il était l'ami du Doyen, et celui-ci avait pensé que s'il n'était pas agrégé de chirurgie, il dirigerait la chaire d'histologie, "tant pis pour Courrier". Mais un autre hasard est intervenu, le voici: les étudiants en droit se mettent en grève parce qu'il n'y avait pas assez de professeurs à la Faculté de Droit d'Alger. Je lis dans le journal que le Directeur général de l'Enseignement supérieur au Ministère va arriver à Alger, pour régler la question de la Faculté de Droit. J'écris aussitôt au Recteur de l'Université d'Alger: “Monsieur le Recteur, apprenant que Monsieur le Directeur général de l'Enseignement supérieur vient à Alger, je vous prie de bien vouloir lui demander de m'accorder une audience". Je ne reçois pas de réponse. Tous les matins, $\mathrm{j}$ 'arrivais au laboratoire assez tôt, or un matin on frappe à la porte, il était $8^{\mathrm{h}} 30$. Je vais ouvrir. Il y avait là le Doyen de la Faculté de Médecine, M. Hérail, le Recteur d'Alger et le Directeur général de l'Enseignement supérieur. Celui-ci s'adressa aussitôt à moi : "Monsieur, vous êtes venu ici pensant être Agrégé, m'apprend le Recteur; vous êtes 'agrégeable'." "Oui, Monsieur le Directeur, d'ailleurs voilà la lettre du Doyen, "Venez à Alger et l'on vous nommera Agrégé d'histologie à la Faculté de Médecine". C'est à la suite de cette lettre que nous sommes venus. Ma femme vient d'être nommée Professeur au lycée d'Alger et moi, je ne suis rien ici".

-Que faites-vous?

-En ce moment, je corrige les épreuves de ma Thèse de Sciences qui sera publiée dans les Archives de Biologie.

- Ah! Ah! vous préparez une Thèse de Sciences?

- La voilà!

- Connaissez-vous un périodique qui s'appelle "Le Bulletin d'Histologie"?

_"Le Bulletin de Policard, oui. Le voici".

Avant d'être au Ministère, il avait été Recteur à Lyon où il avait bien connu Policard qui était professeur d'histologie à la faculté de Médecine de cette ville. Il se tourne alors vers le Doyen et lui dit: "Monsieur le Doyen, c'est le Ministre qui vous parle. Vous avez exactement 48 heures, juste le temps avant mon départ, pour lui trouver une place d'Agrégé". Et je fus nommé Agrégé aussitôt!

Mais je me trouvais tout seul; le chef de travaux allait être nommé Agrégé de 
chirurgie et l'assistante mourait de tuberculose. Je passais mes jours à préparer des cours (trois par semaine pendant toute l'année), à assurer des travaux pratiques (deux séances par semaine, pendant toute l'année) et à faire des préparations pour les travaux pratiques, car celles qui existaient étaient mauvaises. Pendant les vacances je retournais à Strasbourg. En juillet 1927, j'ai passé ma thèse de Sciences là-bas. J'y faisais des préparations et des photographies pour mes étudiants. Mon ami Benoit m'aidait, c'est un admirable technicien, et il avait des inclusions qui étaient excellentes. Il me permettait de tirer une série de cinquante coupes de chacun de ses blocs que je colorais sur place à Strasbourg. J'en tirais des photos pour mon enseignement à Alger. J. Je pense que le rôle de Madame Courrier dans votre vie scientifique a été important. Madame Courrier vous a toujours beaucoup aidé à de nombreux points de vue. Est-ce qu'il vous plairait d'en dire quelques mots?

C. Mais, avec plaisir, et je vous remercie de me poser la question.

Ma femme était agrégée et Professeur d'Histoire naturelle. Pendant longtemps elle m'a traduit les textes anglais; elle n'aime pas parler cette langue, mais elle lit volontiers des livres anglais, en particulier les romans policiers. Son influence sur moi a été très grande, en particulier dans une circonstance spéciale. Un jour un chirurgien d'Alger très connu me fait une proposition: "Mon cher, vous connaissez fort bien la physiologie génitale femelle; moi je suis chirurgiengynécologue. Vous avez un petit traitement d'Agrégé. Nous allons organiser une installation à nous deux: vous ferez l'examen médical et moi je ferai la chirurgie". J'ai demandé son avis à ma femme en lui montrant ce que l'on m'offrait et le changement de vie que cela entraînerait. Elle m'a répondu: "Puisque le laboratoire te plait, eh bien, continue à en faire". Le chirurgien mis au courant de cette décision, s'est écrié: "alors, vous allez vous ennuyer toute votre vie!"'.

During the 12 years that he was to spend at Alger, Courrier and his collaborators carried out a great deal of research on the reproductive physiology of the rabbit, guinea-pig, cat and barbary ape (Macacus inuus).

Courrier had always distinguished two ovarian hormones. Early work by Fraenkel, Loeb and Bouin had caused them to emphasize the rôle of the corpus luteum, whereas Long and Evans and Edgar Allen, concentrating on the vagina of the rat and mouse and discovering 'folliculin' (oestrogen), believed it for some years to be the only ovarian hormone.

C. C'est en mars 1926 que j'ai démontré à la Société de Biologie $(47,62)$, que le liquide folliculaire injecté à forte dose à la lapine castrée était incapable de provoquer la dentelle utérine caractéristique de l'action du corps jaune.

By giving the two hormones in graded amounts, Courrier examined their interactions and inhibitory actions, especially in the rabbit. With $R$. Kehl he studied experimentally the duration (68) and prolongation (73) of the luteal phase of the cycle. Also with Kehl he distinguished between the effects of oestrogens and luteal extracts on the uterine endometrial proliferation (the 'dentelle'), on ovo-implantation and on 
the development of the traumatic deciduoma $(47,58,59,60,80,82$, $95,96,97)$. Both ovo-implantation and the deciduoma could be inhibited by amounts of oestrogen which did not interfere with the 'dentelle', which required less progesterone.

In 1929 (with R. Kehl and R. Raynaud), Courrier made the important observation that the placenta appeared to produce a progestagen which inhibited the normal response of the vagina to oestrogens in ovariectomized, pregnant guinea-pigs $(65,67)$.

D. Pouvez-vous nous parler un peu du placenta? Quand êtes-vous arrivé à la conclusion que le placenta produisait des hormones en quantités importantes?

C. J'avais constaté autrefois que le liquide amniotique humain renfermait beaucoup de folliculine. J'ai fait plus tard des expériences sur la femelle gestante de Cobaye; chez elle à partir d'un certain moment, la castration n'interrompt plus la gestation. Nous avons injecté de la folliculine à des femelles gestantes, castrées, pour observer l'action sur le foetus; nous étudions alors la traversée placentaire de la folliculine. En examinant le vagin de la mère, nous avons constaté qu'il ne réagissait plus à l'hormone folliculaire. Il restait au stade muqueux, le stade du vagin de gestation avec ses grosses assises muqueuses. En augmentant les doses de folliculine, le vagin reste muqueux, on ne produit pas de prolifération (il y a une prolifération normale peu de temps avant l'accouchement, mais elle n'existait pas au moment où nous opérions). Comme nous avions étudié l'antagonisme entre la folliculine et la progestérone, j'ai pensé que l'opposition à la folliculine ne pouvait venir que du placenta en l'absence d'ovaire (65).

A Alger, nous avons beaucoup étudié la Chatte (91, 93, 94, 98, 104, 115, 116), grâce à Gaston Gros et à la Casbah. Nos étudiants allaient à la chasse avec des sardines et nous disposions ainsi de centaines de chats. C'était très pratique. Nous avions à la faculté une vaste cour intérieure bien isolée; j'avais fait construire des cages contre ses murs. Les chattes étaient en liberté dans la cour et les chats mâles étaient enfermés dans les cages. Quand les chattes étaient en oestrus, elles venaient faire les belles devant les cages des mâles et Gaston Gros s'écriait: "celle-là est en rut". On lui ouvrait l'abdomen pour examiner ses ovaires. L'ovulation est provoquée par l'accouplement, la nidation est très tardive, elle se produit le $13^{\mathrm{e}}$ jour après le coït. La réaction utérine de la phase lutéinique est particulière, elle se compose de deux sortes de transformations: dans la première semaine, ce sont les glandes utérines profondes qui se développent d'une façon extraordinaire. Dans la seconde semaine, l'épithélium superficiel forme une dentelle. Quand les blastocystes s'immobilisent dans les cornes utérines, les glandes profondes excrètent et se vident à leur contact.

La castration précoce d'une chatte gestante entraîne, dans certains cas, une dissociation foeto-placentaire (123). Les embryons meurent et restent momifiés sur les placentas; ceux-ci continuent à se développer encore un certain temps pendant lequel la dentelle utérine de cette chatte est maintenue. Il semble bien que le placenta sécrète de la progestérone.

Gaston Gros m'a aidé énormément aussi pour trouver des singes. A cette époque, les singes n'étaient pas chers en Algérie. Les gardes forestiers nous 
signalaient des Arabes qui voulaient bien nous en capturer dans les grottes de Kabylie. J'ai réalisé une dissociation expérimentale des fonctions testiculaires du Macaque en utilisant l'hormone gonadotrope de femme enceinte. Des auteurs avaient constaté que l'urine de femme enceinte contient une hormone gonadotrope. J'ai préparé immédiatement des extraits urinaires renfermant cette hormone gonadotrope et nous les avons injectés à des singes impubères mâles $(106,107,117)$. C'était, je crois, en juin 1934; l'interstitielle se développe d'une façon spectaculaire, mais le tube séminifère reste à l'état impubère; les vésicules séminales et la prostate deviennent énormes. M. Bouin pouvait être satisfait!

Nous avons aussi injecté de la folliculine au jeune singe mâle $(110,111,114)$. Nous avons obtenu une magnifique peau sexuelle comme chez la femelle. C'était la marge de l'anus qui s'oedématiait. Les autres mâles regardaient ce phénomène avec un air perplexe! Et, en même temps, nous avons provoqué une grosse hypertrophie de la prostate; les glandes prostatiques étaient restées au repos complet, c'était le tissu conjonctivo-musculaire situé entre les glandes qui s'était fortement développé. En plus, l'utricule prostatique s'était transformé comme un vagin. Si vous injectez de la folliculine à un chat mâle, l'utricule prostatique réagit de la même manière, mais dans cette espèce, ce sont les glandes prostatiques qui se développent et non le tissu conjonctivomusculaire (139). Pourquoi une telle différence?

En 1934, j'ai décelé la présence de folliculine dans le testicule ectopique de Porc: il y en a beaucoup (109). Zondek, la même année, en a trouvé des quantités dans le testicule de Cheval. En étudiant le testicule ectopique, je voulais démontrer que la folliculine ne provenait pas de la lignée séminale. Malgré la présence de fortes quantités d'oestrogène dans le testicule, il est certain que la testostérone doit normalement dominer puisque l'utricule prostatique d'un mâle normal n'est pas du tout conditionné par la folliculine.

Chez la femelle impubère du singe, l'injection de folliculine détermine bien entendu une hypertrophie considérable de la peau sexuelle (69). Plus tard, nous avons préparé, avec des urines de femmes gestantes, un extrait très concentré de gonadotrope. Il ne renfermait pas trace de folliculine, ainsi que le démontrait le frottis vaginal de la ratte castrée ayant reçu cet extrait. Cette préparation fut administrée à de petites femelles de Macaque âgées d'un an environ; leur puberté survient normalement à 3 ou 4 ans. Nos femelles en expérience étaient donc très éloignées de ce stade. La réaction fut rapide et intense: l'hypertrophie de la peau sexuelle fut le témoin de l'activité ovarienne et de la libération de folliculine. Pendant plus d'un mois, les manifestations génitales externes allèrent en s'accentuant. Mais au bout du $38^{\mathrm{e}}$ jour et malgré la continuation des injections, la peau sexuelle régressa, se plissa, devint exsangue. Après une semaine, apparut un écoulement sanguin vaginal important. La jeune femelle fut sacrifiée à ce moment, chacun des ovaires renfermait un corps jaune vrai: les cellules granuleuses s'étaient lutéinisées. Mais il n'y a pas eu de ponte ovulaire car on découvrit des reliquats d'ovocyte dans le parenchymelutéinique. Celui-ci a été fonctionnel car l'endomètre présentait un aspect menstruel caractéristique. Nous pouvons donc affirmer qu'avec des urines de gestantes, nous avons réalisé, chez une femelle impubère du singe, un cycle ovarien 
complet. La phase folliculaire s'est révélée par les modifications de la peau sexuelle. Celle-ci a régressé au moment de la constitution des corps jaunes; la progestine a exercé son action élective sur l'utérus et ce cycle expérimental s'est terminé par une menstruation vraie. C'était en 1934 (106); il me semble que certains auteurs avaient déclaré que les extraits urinaires humains n'agissaient pas chez le singe.

J. Ce fut une bonne chose que vous ayez eu l'occasion d'étudier un primate, qui, à ce point de vue, ressemble plus à la femme que le rat ou la lapine.

C. Nous avons tenté des expériences de neutralisation de l'action de la progestérone du corps jaune par la folliculine chez la femelle de singe et nous avons été étonnés. Alors qu'il est si facile de neutraliser l'action de la progestérone sur l'utérus de la lapine par l'oestradiol ou par l'oestrone, l'oestrogène ne parait pas capable de s'opposer à l'action de la progestérone sur l'utérus du Macaque. Par contre, cet oestrogène est aisément neutralisé par la progestérone. On le constate sur la peau sexuelle par exemple. En somme, chez le singe c'est la progestérone qui domine (130).

Chez la lapine, avec la proportion $F / P=1 / 75$, c'est la folliculine $(F)$ qui prévaut sur l'utérus; chez le Macaque avec $\mathrm{F} / \mathrm{P}=1 / 6$, la progestérone $(\mathrm{P})$ s'oppose à l'action de l'oestrogène sur la peau sexuelle. Il s'agit certes d'espèces animales et de récepteurs différents.

D. Vous êtes resté 12 ans à Alger?

C. Oui, 12 ans, de 1926 à 1938.

D. Vous aviez espéré partir plus tôt?

G. Non. Je n'y pensais pas; je me plaisais énormément à Alger. J'y travaillais beaucoup, le milieu était très agréable, $\mathrm{j}$ 'avais de bons élèves et quelques étrangers étaient venus au laboratoire pour de longs séjours. Je dois avouer que ma femme et mes deux filles surtout ont été désolées quand nous avons quitté Alger pour Paris.

By 1931, an international standard of pure crystalline oestrone was set up, thanks largely to a $20 \mathrm{~g}$ contribution from M. Girard. Once it was available, Courrier was able to establish that the pure substance could terminate pregnancy in the rabbit (101). Others had maintained that the abortive action of oestrone had been due to impurities, perhaps because other experimental animals (mouse, rat, guinea-pig and cat) tolerated larger doses of oestrogen during pregnancy than the rabbit.

By 1935, crystalline progesterone was also available and Courrier and his collaborators embarked on a long series of experiments, many summarized in a later review (218) on hormone interactions and hormone requirements in different species. Courrier sums up this work in two comprehensive statements.

First:

C. C'est la nature du récepteur sur lequel on travaillait qui a commandé les découvertes. G'est l'étude des récepteurs différents qui a conduit aux deux hormones.

Second: 
C. C'est l'ère lutéinique qui a commencé grâce aux recherches sur l'utérus et sur la gestation. Ce fut ensuite l'ère folliculaire grâce aux recherches sur le vagin. Nous en sommes à l'ère de l'équilibre hormonal et des rapports fonctionnels entre les deux hormones. La chimie est allée plus loin encore puisqu'elle a ouvert l'ère des succédanés artificiels.

In 1937 at the time of the Singer-Polignac Congress in Paris, Professor Jolly invited Courrier to apply for a chair at the Collège de France at Paris. This he was very reluctant to do, since, at Alger, work went well. $\mathrm{He}$ had a laboratory at the hospital as well as at the University and a fine apartment on the bay. Eventually, however, he agreed to submit his name and he was elected to the first chair of Experimental Morphology and Endocrinology, and he gave the inaugural lecture in December 1938.

In 1939, in collaboration with A. Jost, recently arrived in his laboratory, Courrier published a quantitative study of the reproductive endocrinology of the rabbit (143), followed by a paper on hysterectomy in women (144). Endocrinological research continued during and after the war in collaboration with Jost, Bennetz, Poumeau-Delille and later with the physicist, Joliot and others.

Notable papers include those on the androgenic action of pregneninolone in pregnancy $(146,165,166)$, progesterone-oestrogen synergism affecting the pubic symphysis of guinea-pigs (151, 157, 160), and especially on the survival of extra-uterine fetuses in the ovariectomized rabbit (152). Other papers recorded the local antagonistic action of oestrogen on the pseudopregnant rabbit uterus (173), and local interactions of oestrogen and progesterone on the mouse vagina (178).

C. Nous avons étudié aussi avec A. Jost, la synergie oestro-progestéronique. Chez la lapine gestante, la castration provoque toujours l'avortement. Une apine gestante castrée, qui reçoit $5 \mathrm{mg}$ de progestérone par jour, fait une gestation normale, on peut trouver par exemple neuf foetus en fin de gestation. Si l'on injecte seulement $0.5 \mathrm{mg}$ de progestérone par jour, l'avortement se produit. Mais si, à ces $0.5 \mathrm{mg}$ de progestérone, on ajoute des quantités minimes d'oestradiol ( $0.66 \mu \mathrm{g}$ par jour), la gestation se poursuit, il y a donc synergie hormonale.

Nous avons obtenu aussi une dissociation foeto-placentaire: on castre une lapine au $4^{e}$ jour de la gestation, et on lui injecte $2 \mathrm{mg}$ de progestérone par jour, on découvre plus tard huit placentas bien développés avec de petits embryons momifiés sur les placentas; c'est une belle dissociation. En somme l'insuffisance lutéinique peut conduire à la dissociation foeto-placentaire, ou à des gestations partielles avec côte à côte des foetus vivants et des foetus morts. On peut observer aussi des foetus anormalement petits et certaines anomalies: spina bifida, anencéphalie etc. . (221, 222). Semblables anomalies existent parfois chez le foetus humain. S'agit-il aussi d'une déficience lutéinique?

En juin 1941, j'ai présenté à la Société de Biologie une note sur l'évolution de la grossesse extra-utérine expérimentale chez la lapine castrée (152). Nous avons enregistré depuis quelques rares confirmations; mais les succès sont très 
difficiles à obtenir. Nous avions provoqué une gestation unilatérale par ligature tubaire. Au $19^{e}$ jour, castration. Il y a trois foetus dans la corne gravide, j'en extériorise un, en laissant le placenta in situ, j'enlève simplement le muscle utérin pour libérer la cavité amniotique. A la fin de la gestation, juste avant l'accouchement, que trouve-t-on? Le placenta du foetus extériorisé est allé se greffer sur le péritoine, ce foetus est vivant, de taille normale. C'est extraordinaire de voir que le placenta s'est décollé de l'utérus, a été se fixer sur le péritoine et que le foetus a une taille normale pour la fin de la gestation. Les deux autres foetus que j'avais laissés dans l'utérus ont été retrouvés en décomposition dans le ventre de la mère, ils avaient environ la taille du $20^{\circ}$ jour. Ils avaient été expulsés par le trou de celui qui s'était détaché. Il semble qu'en l'absence d'hormone ovarienne, l'utérus se rétracte et ne peut plus suivre l'expansion foetale. Les foetus intra-utérins paraissent écrasés.

Nous avons essayé aussi chez la ratte la gestation extra-utérine. Nous avons commencé par la castration au $12^{\mathrm{e}}$ ou au $13^{\mathrm{e}}$ jour. On peut observer par la suite des foetus vivants, mais ils sont très comprimés avec des ecchymoses et des déformations. Dans une autre expérience, après avoir castré, j’ai libéré un sac embryonnaire pour provoquer une grossesse extra-utérine. Les foetus laissés dans l'utérus sont comprimés, les uns morts, les autres encore vivants. J'ai obtenu une grossesse extra-utérine chez la ratte de cette façon en décembre 1949.

Passons maintenant à l'étude de la prégnéninolone. Dans cette même molécule existent trois actions hormonales différentes. En octobre 1938, je reçois une lettre de Hohlweg qui avait préparé avec Inhoffen un stéroïde artificiel appelé prégnéninolone ou éthinyltestostérone. Il lui avait reconnu une activité progestative en l'administrant par la bouche; il avait provoqué ainsi la dentelle utérine de la lapine. Mais il n'arrivait pas avec cette substance à maintenir la gestation chez une lapine castrée. Or pour moi, c'est le meilleur critère pour affirmer qu'une substance est bien une substance progestative vraie. Du premier coup, avec des doses très fortes, nous avons obtenu, Jost et moi, la nidation et le maintien de la gestation chez la lapine castrée (145). Mais il faut commencer à donner le produit 24 heures environ avant la castration. En avril 1939, nous constatons que la prégnéninolone ou éthinyltestostérone $(159,171)$ exerce une action androgène importante chez le rat mâle castré (146), ce qui n'est pas le cas pour la progestérone. En 1942, nous remarquons que les foetus femelles de la lapine gestante traitée par la prégnéninolone, sont masculinisés $(165,166,171,175)$. De même que la folliculine, la prégnéninolone administrée à la mère traverse le placenta et agit sur les foetus (323). Les industries ont cependant continué à fabriquer de la prégnéninolone, en l'appelant 'le progestatif buccal'. La prégnéninolone est peut-être androgène chez la lapine, nous disait-on mais elle ne l'est sûrement pas chez la femme. Seize ans après, Wilkins, de Baltimore, signala des anomalies génitales chez les filles nouveau-nées, dont les mères, en menace d'avortement, avaient pris des comprimés de prégnéninolone. Je dois signaler que pour ces expériences, c'est Jost qui a fait les coupes et qui a découvert l'action androgène sur le foetus femelle.

Retenons de cette sombre histoire que les résultats des expériences faites sur les animaux ont parfois une certaine importance en médecine humaine. 
From 1944 Courrier returned to the thyroid, collaborating with Joliot, and others on radioactive thyroxin and various aspects of the metabolism of the gland $(179,181,190,191,215,216,226,227,242,249,251,253$, $259,270,272,272,273,296)$. They showed that thyroxin at relatively weak doses could prevent radioactive iodine from penetrating the thyroid.

G. Je suis revenu à la thyrö̈de parce que dès mon arrivée au Collège de France, j'avais rencontré $F$. Joliot qui, avec sa femme Irène Joliot-Gurie, avait découvert la radioactivité artificielle. Il possédait dans son laboratoire du Collège un cyclotron, le premier cyclotron d'Europe à cette époque. Je lui ai demandé de l'iode radioactif et comme il s'intéressait beaucoup à mes premières recherches sur la thyroïde, nous avons travaillé ensemble. J'ai compris très vite pourquoi les animaux soumis à l'alimentation thyroïdienne avaient une thyroïde au repos. Nous avons constaté en effet que chez le Rat traité à la thyroxine, l'iode radioactif ne pouvait plus pénétrer dans la thyroïde. De plus, mon collaborateur A. Horeau fabriqua de la thyroxine radio-active et celle-ci se concentrait dans l'hypophyse $(216,226)$.

J'ajouterai que ces recherches venaient de commencer quand survint la guerre. L'évolution de celle-ci fut telle qu'un jour une équipe de physiciens allemands en uniforme arriva au Collège, attiré par notre précieux cyclotron. C'est alors que Joliot entreprit un travail clandestin afin que l'appareil auquel il tenait tant fonctionne le plus mal possible.

In 1945 came the publication of the book on which he had been working during the war, Endocrinologie de la Gestation, the first comprehensive study of the field and a great stimulus to future research (193). This was to be followed in 1955 by a further review (266, in collaboration with $M$. Baclesse). In 1950, Courrier summarized in English much of his work on the interactions of oestrogen and progesterone (219).

Courrier was always interested in the multiple properties of steroid hormones and he experimented with purified synthetic and radioactive hormones as they became available $(182,183,184,192,196,199,200$, $202,204,205,208,210,211,213,223,232)$. He also investigated the progestational action of desoxycorticosterone and later the properties of other corticosteroids and their relation to reproductive physiology (149, $161,162,163,170,228,230,236,237,241,247,248,305)$.

Between 1956 and 1964, Courrier and his collaborators published on hypophysial grafts in rats $(273,282,285,293,295,297,303)$ and on the purification of hypophysial hormones from sheep pituitaries (294, 306, $307,315,321,326,327)$. They showed that hypophysial kidney grafts could maintain spermatogenesis, interstitial cell activity and testis weight in hypophysectomized rats, although such grafts were almost devoid of TSH or ACTH activity. These results contrasted with those of other workers who had concluded that the hypophysis could only function in contact with the hypothalamus.

Other problems existed in connection with the restoration of testis function, in particular the hormonal control of spermatogenesis and 
interstitial cell activity. There was evidence of the effect of androgens in temporarily maintaining spermatogenesis after hypophysectomy but Courrier's team showed that highly purified FSH (LH contamination less than $1 \%$ ), acting alone, could induce spermatogenesis 2 to 4 weeks after hypophysectomy, while the interstitial tissue, responsive to $\mathrm{LH}$, showed hardly any reaction $(326,333)$. This demonstration of the almost complete independence of tubules and interstitial cells in the testis confirmed Courrier's earlier work, as did his final study of a transplantable interstitial cell tumour, free from tubules, implanted first in castrated immature rats and later in hypophysectomized males, where it continued to secrete androgens but did not grow (328, $330)$.

J. Quand M. Courrier a été nommé Professeur au Collège de France en 1938, je crois que c'est la seule chaire en France qui portait le titre d'Endocrinologie. Elle s'appelait 'Morphologie expérimentale et Endocrinologie'.

C. Il est certain que la morphologie expérimentale eut une grande importance en endocrinologie sexuelle.

D. Je voudrais vous demander de parler un peu du Collège de France.

G. Le Collège de France a été la première institution libérale en France. Il a

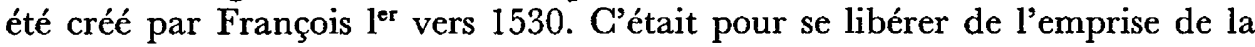
Sorbonne dont l'enseignement officiel était scholastique.

J. Elle était dominée par la Faculté de Théologie, la Sorbonne.

C. Certainement. François $1^{\text {er }}$, sous l'influence de son bibliothécaire, Guillaume Budé, a créé le Collège de France pour y nommer des personnalités qui enseigneraient des choses importantes et originales. On avait demandé à Erasme, qui était aux Pays-Bas, d'accepter une chaire au Collège de France. "Merci bien", répondit-il, "je n'ai pas envie d'être brûlé par les gens de la Sorbonne".

Au début on enseignait surtout les langues: le Latin, le Grec, l'Hébreu. Mais très tôt furent nommés des professeurs de Mathématiques, puis des médecins. Il est intéressant de signaler qu'au moment de la Révolution, les Universités et les Facultés ont été fermées, tandis que le Collège de France fut respecté par les Révolutionnaires.

Sa devise était "Docet omnia". Au Collège, les chaires ne sont pas permanentes. Quand une chaire est vacante, son titulaire ayant disparu, soit par décès, soit par mise à la retraite, c'est un 'crédit' qui est vacant et non pas une discipline. Les Professeurs du Collège de France se réunissent pour décider que faire de ce crédit vacant. Ils s'efforcent de trouver une science de 'pointe', mais à condition d'avoir pour parler de cette science un homme de 'pointe'. Ce qu'on recherche à la fois, c'est une science en pleine expansion et un titulaire qui participe à cette expansion. L'élection se fait en deux temps: dans un premier temps, on vote pour l'utilisation du crédit, c'est-à dire pour un titre de chaire. A ce moment-là, il y a déjà, derrière les titres de chaires proposés, des candidats car ils ont fait des visites, on les connait. Mais on ne parle pas encore officiellement des canditats, on défend seulement l'intérêt des chaires proposées. Ainsi la susceptibilité des candidats futurs est respectée. 
D. G'est au candidat de se présenter?

C. Oui, mais on le lui demande.

D. Il faut une majorité des deux tiers?

C. Non, la majorité absolue: la moitié plus un. Quand un certain titre de chaire a obtenu cette majorité, le Collège demande au Gouvernement de déclarer cette chaire vacante. C'est alors que le candidat officieux-celui qui était derrière ce titre-devient candidat officiel. Il pose sa candidature à la nouvelle chaire. Il est sûr d'être élu sauf des cas exceptionnels. Ce sont, vous le constatez, des titres de chaires qui sont en concurrence.

J'ajouterai que les cours du Collège de France sont publics, ouverts gratuitement à tout le monde. Le Professeur doit répondre aux questions qui lui sont posées après sa leçon.

D. Et après votre chaire au Gollège de France vous devenez Secrétaire perpetuél de l'Académie des Sciences. Voulez-vous en parler?

G. L'Académie des Sciences a été fondée en 1666. Son tricentenaire fut célébré avec éclat en 1966. Elle est une des cinq Académies qui constituent l'Institut de France, les quatre autres étant l'Académie française, l'Académie des Inscriptions et Belles lettres, l'Académie des Beaux-Arts, l'Académie des Sciences morales et politiques. Votre grand Churchill était membre associé étranger de celle-ci; en prenant séance, il a déclaré en souriant qu'il venait faire la jonction entre la morale et la politique.

L'Institut de France est un organisme d'Etat, ses membres ont un costume d'apparat que l'on appelle 'l'habit vert' parce qu'il est couvert de broderies de soie verte; ils reçoivent une indemnité académique très faible.

L'Académie des Sciences est en relation avec les Unions scientifiques internationales et avec les Académies de nombreux pays. Elle se réunit une fois par semaine: le lundi à 15 heures. Au cours de sa séance, elle accueille les savants étrangers de passage à Paris, elle prend connaissance des ouvrages qui lui sont adressés, elle entend les communications présentées par ses membres. Elle reçoit aussi de nombreuses notes envoyées par des auteurs qui ne sont pas Académiciens. Mais ces notes doivent être transmises par des membres de l'Académie qui en sont responsables. Les notes admises sont publiées dans les Comptes Rendus de l'Académie des Sciences; la publication est très rapide, elle se fait en 15 jours. Les Comptes Rendus comprennent quatre séries: Mathématique, Physique, Chimie, Sciences naturelles.

Le nombre des membres de l'Académie des Sciences est fixé, actuellement nous sommes 100; ce nombre est environ le double de celui des autres Académies de l'Institut de France. En plus de ces 100 membres titulaires, il existe aussi des Associés étrangers et des Correspondants.

L'administration de chacune des cinq Académies est dirigée par un Secrétaire perpétuel choisi parmi les membres. Comme l'Académie des Sciences est la plus nombreuse, elle possède deux Secrétaires perpétuels: le premier est $M$. le duc Louis de Broglie qui s'occupe de la première division (Sciences mathématiques et physiques); le second est moi-même pour la deuxième division (Sciences chimiques et naturelles). Chacune des deux divisions comprend des sections pour les diverses disciplines. Il existe en outre la division des membres libres réservée en principe aux Sciences nouvelles. 
Les Académies possèdent des biens propres, les revenus de leurs fondations sont destinés à donner des prix.

D. Je vois que vous avez beaucoup à faire!

C. Tous les ans, en novembre et décembre, chacune des cinq Académies se réunit sous la fameuse Coupole de l'Institut. A cette Séance solennelle, les membres se présentent en habit vert avec l'épée au côté. Le Président fait l'éloge des disparus au cours de l'année. On donne lecture du palmarès des lauréats. Le Secrétaire perpétuel lit une Notice sur la vie et l'oeuvre d'un membre décédé particulièrement célèbre. L'an dernier, j'ai parlé de Cuvier parce que c'était le bicentenaire de sa naissance; j'ai naturellement insisté sur des faits peu connus de sa vie dont on trouve la relation dans nos archives qui sont très riches.

Courrier's obituaries of deceased academicians and others were prepared with great care and are valuable sources of information (353).

D. Je vous remercie beaucoup M. Courrier.

C. Mais c'est moi qui suis très honoré, car vous avez décidé de venir à Paris pour me rajeunir en m'interrogeant sur ma vie et sur mes premières recherches.

\section{GHRONOLOGIGAL LIST OF PUBLICATIONS}

\section{0}

1. Sur l'existence d'une sécrétion épididymaire chez la Chauve-souris hibernante; sa signification. C. R. Séanc. Soc. Biol. 83, 67.

2. Sur l'existence d'une sécrétion de l'épithélium utérin chez la Chauve-souris hibernante; sa signification. C. r. Séanc. Soc. Biol. 83, 243.

\section{1}

3. Action sur le thymus de l'ingestion de glande thyroïde. C. r. Séanc. Soc. Biol. 84, 226.

4. Contribution à l'étude morphologique et fonctionnelle de l'epithélium du pavillon de l'oviducte chez les Mammifères. C. r. Séanc. Soc. Biol. 84, 571.

5. Sur le rôle physiologique des sécrétions utérines et tubaires chez la Chauve-souris hibernante. $C$. r. Séanc. Soc. Biol. 84, 572.

6. Un Schizotrypanum chez la Chauve-souris en Basse-Alsace. Schizotrypanose et goître endémique. (with E. Chatton.) C. r. Séanc. Soc. Biol. 84, 943.

7. Sur un trypanosome de la Chauve-souris: Vesperugo pipistrellus, à formes crithidiennes intratissulaires et cystigènes. Hypothèse relative à l'étiologie du goître endémique. (with $\mathrm{E}$. Chatton.) C. r. hebd. Séanc. Acad. Sci., Paris, 172, 1254.

8. Glande interstitielle du testicule et caractères sexuels secondaires chez les Poissons. C. r. hebd. Séanc. Acad. Sci., Paris, 172, 1316.

9. Action de l'ingestion de corps thyroïde sur la glande germinative mâle. C. r. Séanc. Soc. Biol. 85, 484.

10. Sur le conditionnement des caractères sexuels secondaires chez les Poissons. C. r. Séanc. Soc. Biol. $85,486$.

11. Sur le déterminisme des caractères sexuels secondaires chez les Arthropodes. C. r. hebd. Séanc. Acad. Sci., Paris, 173, 668.

12. Sur l'existence d'une sécrétion intra-nucléaire dans l'épithélium du spermathèque de la Reine d'Abeille; sa signification. C. r. Séanc. Soc. Biol. 85, 941.

13. Sur l'existence d'une glande interstitielle dans le testicule des Poissons. C. r. Séanc. Soc. Biol. 85, 939. 
1922

14. Sur l'indépendance de la glande séminale et des caractères sexuels secondaires chez les Poissons; étude expérimentale. C. r. hebd. Séanc. Acad. Sci., Paris, 174, 70.

15. Appareil réticulé de Golgi et polarité sécrétoire des cellules parathyroïdiennes. (with P. Reiss.) C. r. Séanc. Soc. Biol. 86, 867.

16. Gontribution à l'histophysiologie du corps thyroïde. C. r. Séanc. Soc. Biol. 86, 869.

17. Etude préliminaire du déterminisme des caractères sexuels secondaires chez les Poissons. Archs Anat. Histol. Embryol. 1, 115-144.

18 Démonstration sur la glande interstitielle du testicule et les caractères sexuels secondaires chez l'Epinoche. Ass. Anatomistes, Gand, 1922.

19. Le cycle glandulaire de l'épithélium de l'oviducte chez la Chienne. (with H. Gerlinger.) C. $r$. Séanc. Soc. Biol. 87, 1363.

20. Le cycle génital de la femelle chez certains Mammifères hibernants. C. r. Séanc. Soc. Biol. 87, 1365.

21. Sur l'existence d'une glande interstitielle dans le testicule des Blennies. Bull. Soc. zool. Fr. 47, 459.

\section{3}

22. Action d'un colloïde électrolytique, le Rouge Congo, sur l'oeuf d'Oursin (Paracentrotus lividus). C. r. Séanc. Soc. Biol. 88, 196.

23. La structure de l'épithélium du vagin chez le Gobaye et ses modifications. C. r. Ass. Anat. Lyon. 145.

24. Remarque sur la membrane de fécondation de l'œuf d'Oursin (Paracentrotus lividus). C. r. hebd. Séanc. Acad. Sci., Paris, 176, 1260.

25. Cycle annuel de la glande interstitielle du testicule chez les Cheiroptères. Coexistence du repos séminal et de l'activité génitale. C. r. Séanc. Soc. Biol. 88, 1163.

26. Vésicule blastodermique parthénogénétique dans un ovaire de Cobaye impubère. Archs Anat. Histol. Embryol. 2, 453.

27. Sur l'apparition de graisse osmiophile au cours du cycle sécrétoire de certaines cellules glandulaires, sa signification. C. r. Séanc. Soc. Biol. 89, 589.

28. Métaplasie osseuse au niveau d'ovaires greffés dans le testicule chez le Cobaye. Bull. Soc. Anat. Paris.

29. Formation d'un complexe xéno-parasitaire geant avec bordure en brosse sous l'influence d'une Microsporidie dans le testicule du Cottus bubalis. (with E. Chatton.) C. r. Séanc. Soc. Biol. 89, 579.

30. Parthénogenèse spontanée dans l'ovaire de Cobaye. (with Ch. Oberling.) Bull. Soc. Anat. Paris.

31. Un trypanosome nouveau des Chauves-souris de la Cathédrale de Strasbourg. (with E. Chatton.) Bull. Ass. philomath. Als. Lorr. 6, 272.

32. Le cycle de la glande interstitielle et l'évolution des caractères sexuels secondaires chez les Mammifères à spermatogénèse périodique. C. r. Séanc. Soc. Biol. 89, 1311.

\section{4}

33. Nouvelles remarques sur la membrane de fécondation de l'œuf d'Oursin (Paracentrotus lividus). C. r. hebd. Séanc. Acad. Sci., Paris, 178, 249.

34. Perméabilisation expérimentale de l'œuf vierge d'Oursin (Paracentrotus lividus). C. r. hebd. Séanc. Acad. Sci., Paris, 178, 597.

35. Rut expérimental chez la femelle castrée et chez la femelle impubère. C. r. Séanc. Soc. Biol. 90, 453.

36. Le rythme vaginal du Hérisson, action de l'injection de liquide folliculaire. C. r. Séanc. Soc. Biol. 90, 808.

37. Sur la structure particulière de la prostate externe du Hérisson. C. r. Ass. Anat. Strasbourg, p. 109.

38. Nouvelles recherches sur la folliculine. Contribution à l'étude du passage des hormones au travers du placenta. C. r. hebd. Séanc. Acad. Sci., Paris, 178, 2192.

39. Le cycle sexuel chez la femelle des Mammifères. Etude de la phase folliculaire. Thèse de Médecine, Strasbourg. In Archs Biol. 34, 369.

40. Réaction histologique du corps thyroïde des animaux soumis à l'alimentation thyroïdienne. $C . r$. Séanc. Soc. Biol. 91, 1274.

41. Les caractères sexuels secondaires et le cycle testiculaire chez l'Epinoche. Reply to G.-J. van Oordt. Archs Anat. Histol. Embryol. 4, 471.

42. Les hormones ovariennes. Revue fr. Endocr. 3, 94.

43. Démonstration sur les réactions du corps thyroïde des animaux soumis à l'alimentation thyroïdienne. C. r. Ass. Anat. Turin, p. 427.

44. Le déterminisme de la sécrétion tubaire. C. r. Séanc. Soc. Biol. 93, 672.

45. Nymphomanie et ovaires kystiques. C. r. Séanc. Soc. Biol. 93, 674. 
46. Modifications vaginales chez la Lapine au cours de la vie génitale. C. r. Séanc. Soc. Biol. 94, 280.

47. Réaction utérine chez la Lapine castrée à l'injection de liquide folliculaire. (with R. Potvin.) C. r. Séanc. Soc. Biol. 94, 878.

48. La dégénérescence wallérienne chez les Chauves-souris hibernantes. C. r. Séanc. Soc. Biol. 94, 1385.

49. Les effets de la castration chez les Chéiroptères. C. r. Séanc. Soc. Biol. 94, 1386.

50. Un cas d'eunuchoïdisme avec spermatogenèse normale chez une Pipistrelle. C. r. Ass. Anat. Liège, p. 176.

51. Sur l'action quantitative de l'hormone folliculaire. C. r. hebd. Seanc. Acad. Sci., Paris, 182, 1492.

1927

52. Etude sur le déterminisme des caractères sexuels secondaires chez quelques Mammifères à activité testiculaire périodique. Thèse de doctorat è Sciences naturelles, Strasbourg. In Archs Biol. 37, 173.

53. Les données récentes sur l'histophysiologie ovarienne. Rapport. présenté à l'A.F.A.S., Congrès de Constantine, p. 31 .

54. Pseudo-grossesse créee par la persistance d'un corps jaune périodique. (with H. Duboucher \& E. Pouget.) C. r. Séanc. Soc. Biol. 97, 271.

1928

55. Action de l'ingestion de corps thyroïde sur le thymus, sur le testicule et sur la thyroïde-Contribution à l'histophysiologie thyroïdienne. Revue fr. Endocr. 6, 10.

56. Sur l'existence d'une crise génitale du nouveau-né chez le Cobaye. C. r. Séanc. Soc. Biol. 99, 24.

57. La présence du follicule mûr n'est pas indispensable à l'apparition de l'œestrus. C. r. Séanc. Soc. Biol. $99,26$.

58. L'hormone folliculaire inhibe-t-elle l'hormone lutéinique? C. r. Séanc. Soc. Biol. 99, 224.

59. Des rapports qui existent entre les hormones folliculaire et lutéinique. (with R. Masse.) C. r. Seanc. Soc. Biol. 99, 263.

60. La folliculine ne peut suppléer l'hormone lutéinique. (with R. Masse.) C. r. Séanc. Soc. Biol. 99, 265.

61. Insuline et folliculine. C. r. Séanc. Soc. Biol. 99, 1630.

62. Nouvelles recherches sur les hormones ovariennes. Unicisme ou dualisme. Bull. Histol. appl. Physiol. Path. 5, 394.

63. Sur la constatation de parasites dans des diverticules multiples du jéjunum. (with P. Goinard.) Annls Anat. path. 6, 189.

64. Sur le mode d'action des extraits hypophysaires antérieurs. (with R. Kehl.) C. r. Séanc. Soc. Biol. 100, 711.

65. Existe-t-il un mécanisme neutralisant l'hormone folliculaire chez la femelle gestante castrée? (with R. Kehl \& A. Raynaud.) C. r. Ass. Anat. Bordeaux, p. 166.

66. Sur le passage de l'hormone thyroïdienne de la mère au foetus à travers le placenta. (with M. Aron.) C. r. Séanc. Soc. Biol. 100, 839.

67. Neutralisation de l'hormone folliculaire chez la femelle gestante castrée. (with R. Kehl \& A. Raynaud.) C. r. Seanc. Soc. Biol. 100, 1103.

68. Sur la durée de l'activité lutéinique pendant la gestation. (with R. Kehl.) C. r. Séanc. Soc. Biol. 101, 345.

69. Action des extraits hypophysaire et folliculaire chez la Guenon impubère. (with R. Kehl \& A. Raynaud.) C. r. Séanc. Soc. Biol. 101, 1093.

70. Les modifications saisonnières de l'appareil uro-génital chez Uromastix acanthinurus (Bell). Archs Anat. microsc. 25, 388.

71. La greffe des cornes utérines chez Ia Lapine; leurs réactions aux hormones génitales. (with P. Bouin.) Archs Anat. microsc. 25, 189.

\section{0}

72. Structure et histophysiologie de l'appareil génital femelle. Encyclopédie médico-chirurgicale, Gynécologie $6,17$.

73. Etude de la réaction utérine au cours d'une phase lutéinique artificiellement prolongée. (with R. Kehl.) Alger. méd. pp. 1-4.

74. L'atrophie de la thyroide provoquée par l'opothérapie thyroïdienne. Notion d'un équilibre glandulo-humoral. Archs fr.-belg. Chir. 32, (1).

75. Traversée placentaire de la folliculine et crise génitale chez le Gobaye nouveau-né. C. r. Ass. Physiol Alger.

76. L'antagonisme entre les hormones ovariennes. Ass. fr. Avanc. Sciences, Alger, p. 383. 
77. Recherches sur l'antagonisme des hormones folliculaire et lutéinique. C. r. Séanc. Soc. Biol. 104, 280.

78. La folliculine, injectée à fortes doses, a-t-elle une action sur l'ovisac en maturation? C. r. Séanc. Soc. Biol. 104, 282.

79. Folliculine et phénomènes utérins préparatoires à la nidation de l'œuf. C. r. Séanc. Soc. Biol. 104, 1178.

80. Le déciduome expérimental chez la Lapine gestante. (with R. Kehl.) C. r. Séanc. Soc. Biol. 104, 1180.

81. Recherches sur le mécanisme de la crise génitale du nouveau-né. 2nd Int. Congr. Sex Res., London, p. 352.

82. Action de la folliculine sur les modifications de la phase lutéinique. (with R. Kehl.) 2nd Int. Congr. Sex Res., London, p. 357.

\section{1}

83. Les bases de l'opothérapie ovarienne. Fédn Soc. Sci. méd. Alger.

84. Les hormones sexuelles femelles. C. r. Séanc. Soc. Biol. 107, 1367.

85. Nouvelles recherches sur l'action de la folliculine chez la Lapine en phase lutéinique. (with $\mathbf{R}$. Kehl.) C. r. Séanc. Soc. Biol. 107, 1547.

86. Sur les gaines périvasculaires de la muqueuse utérine chez la Lapine. (with R. Kehl.) C. r. Ass. Anat. Varsovie, p. 144.

87. Notes d'endocrinologie ovarienne. Presse med. 92.

\section{2}

88. Sur la suspension expérimentale de la phase lutéinique. (with R. Kehl.) C. r. Séanc. Soc. Biol. 109, 877.

89. Modifications génitales chez les animaux à croissance arrêtée par le déficience en lysine. (with R. Raynaud.) C. r. Séanc. Soc. Biol. 109, 881.

90. Notes on ovarian endocrinology. Am. Med. 38, 105.

91. Contribution à l'étude du cycle génital chez la Chatte. (with G. Gros.) C. r. Séanc. Soc. Biol. 110, 51.

92. Dualisme humoral ovarien et antagonisme des hormones ovariennes. Liege méd. 27, 861.

93. Grise génitale expérimentale chez la Chatte nouveau-née. (with G. Gros.) C. r. Séanc. Soc. Biol. $110,1021$.

94. Remarques sur la nidation de l'œuf chez la Chatte. (with G. Gros.) C. r. Seanc. Soc. Biol. 111, 787.

\section{3}

95. Sur l'avortement folliculinique chez la Lapine. (with R. Kehl.) C. r. Seanc. Soc. Biol. 112, 675.

96. Nouvelles recherches sur la gestation chez la Lapine. (with R. Kehl.) C. r. Séanc. Soc. Biol. 112, 940.

97. Sur l'existence de seuils différentiels endocriniens dans les réactions utérines de la phase lutéinique. (with R. Kehl.) C. r. Séanc. Soc. Biol. 113, 607.

98. Données complémentaires sur le cycle génital de la Chatte. (with G. Gros.) C. r. Séanc. Soc. Biol. 114, 275.

99. Contribution à l'endocrinologie de la gestation chez la Lapine. (with R. Kehl.) C. r. Séanc. Soc. Biol. 114, 1317.

100. Sur le cas d'une Poule de race bédouine qui pondit exclusivement des œufs nains, privés de jaune. (with J. Benoit.) C. r. Séanc. Soc. Biol. 114, 1335.

101. Expériences d'antagonisme humoral ovarien réalisées avec l'étalon international de folliculine cristallisée. (with R. Raynaud.) C. r. Séanc. Soc. Biol. 115, 299.

1934

102. Cancer et acides aminés indispensables à la croissance. (with G. Coste.) C. r. Séanc. Soc. Biol. 115, 631.

103. Action des substances urinaires gonadotropes chez la Marmotte hibernante. (with G. Gros.) C. r. Séanc. Soc. Biol. 115, 1097.

104. Démonstration sur le cycle utérin chez la Chatte. (with G. Gros.) C. r. Ass. Anat. Bruxelles, p. 550.

105. Etude quantitative de l'avortement folliculinique provoqué chez la Lapine par l'hormone cristallisée. Réalisation d'un avortement partiel. (with R. Raynaud.) C. r. Séanc. Soc. Biol. 116, 1073.

106. Action des substances urinaires gonadotropes chez la femelle impubère du Singe. (with G. Gros.) C. r. Séanc. Soc. Biol. 116, 1392.

107. Action des substances urinaires gonadotropes chez le Singe mâle impubère. Etude cytologique de la réaction diastématique. (with G. Gros.) C. r. Séanc. Soc. Biol. 116, 1396.

108. A propos du livre récent de E. F. Terroine sur le métabolisme de l'Azote. Revue fr. Endocr. 12, 398.

109. Recherches sur la teneur en substance cestrogène des testicules normaux et ectopiques. $C . r$. Séanc. Soc. Biol. 117, 1117. 
110. Action de la folliculine chez le Singe mâle impubère.-1. Apparition d'une peau sexuelle. (with G. Gros.) C. r. Seanc. Soc. Biol. 118, 683.

111. Action de la folliculine chez le Singe mâle impubère.-2. Modifications des annexes. (with G. Gros.) C. r. Séanc. Sac. Biol. 118, 686.

1935

112. L'antagonisme hormonal ovarien. Alger. méd. 86, 57, and Bull. Hist. appl. Physiol. Path. 12, 261.

113. Sur les rapports fonctionnels des hormones ovariennes. Gynéc. Obstet. 31, 835.

114. Réaction du Magot mâle impubère à l'hormone ostrogène. (with G. Gros.) Gynéc. Obstet. 31, 845.

115. Action de la folliculine chez la Chatte gestante. (with G. Gros.) C. r. Séanc. Soc. Biol. $120,8$.

116. Contribution à l'endocrinologie de la grossesse chez la Chatte. (with G. Gros.) C. r. Séanc. Soc. Biol. 120,5 .

117. Notes d'endocrinologie sexuelle chez le Singe d'Algérie. Brux. méd. 18, 674.

118. Notes d'endocrinologie génitale femelle. (with R. Kehl.) Transactions on the dynamics of development, Moscow, 10, 59.

119. Sur une nouvelle méthode de dosage biologique des extraits de corps jaune. C. r. Séanc. Soc. Biol. $120,1263$.

120. Les réactions du Singe mâle impubère à certaines substances hormonales. (with G. Gros.) Bull. Soc. Hist. nat. Afr. $\mathcal{N}$. 26, bis, 150.

1936

121. L'utricule prostatique chez le Cobaye soumis à la folliculinisation. (with G. Cohen-Solal.) C. $r$. Séanc. Soc. Biol. 121, 903.

122. Hormones et tumeurs. Alger. méd. p. 71.

123. Dissociation foeto-placentaire réalisée par la castration chez la Chatte. (with G. Gros.) C. r. Séanc. Soc. Biol. 121, 1517.

124. Sur l'unité nidatrice de progestine. Archs port. Sci. biol. 5, 173.

125. Les hormones féminines en thérapeutique. Vie méd. p. 253.

126. Etude quantitative de l'antagonisme humoral ovarien effectuée avec les deux hormones cristallisées. C. r. Séanc. Soc. Biol. 122, 661.

127. Les hormones ovariennes. Annls. Bull. Soc. r. Méd. Gand, 14, 121 and Revue méd. Paris.

\section{7}

128. Etude quantitative de l'action antiœstrale de la progestine à l'aide des deux hormones cristallisées. (with G. Gohen-Solal.) C. r. Séanc. Soc. Biol. 124, 961.

129. Sur les rapports des hormones mâle et femelle: testostérone et folliculine. Etude quantitative de leur antagonisme. (with G. Cohen-Solal.) C. r. Séanc. Soc. Biol. 124, 925.

130. Etude des rapports fonctionnels entre les hormones ovariennes chez les Primates. (with G. Gros.) C. r. Séanc. Soc. Biol. 125, 746.

131. Les rapports fonctionnels des hormones ovariennes. Colloque int de la Fondation Singer-Polignac. Hermann, Paris.

132. Biologie des hormones sexuelles femelles. Journées méd. int. Paris, p. 328. Baillière, Paris.

133. Sur les variations de l'histophysiologie génitale femelle. Soc. Roumaine Bull. Mém. Section. Endocr. p. 217. and Gaz. méd. Fr. p. 829.

134. Sur les corrélations fonctionnelles des deux hormones de l'ovaire. (with R. Kehl.) C. r. Séanc. Soc. Biol. 127, 140.

135. Données préliminaires sur le besoin quantitatif en progestine chez la Lapine pleine castrée. Realisation de grossesses partielles. (with R. Kehl.) C. r. Séanc. Soc. Biol. 127, 529.

1938

136. Influence du propionate de testostérone sur l'utérus. (with G. Gros.) C. r. Séanc. Soc. Biol. 127, 921.

137. Sur le besoin hormonal quantitatif chez la Lapine gestante castrée. (with R. Kehl.) C. r. Séanc. Soc. Biol. 128, 188.

138. Nouvelles recherches sur l'action du propionate de testostérone chez la femelle. (with G. Gros.) C. r. Séanc. Soc. Biol. 128, 194.

139. Sur l'action de la folliculine chez le Chat mâle. (with G. Gros.) C. r. Séanc. Soc. Biol. 129, 8.

140. Physiologie du sexe. (with R. Kehl.) Revue annuelle de Physiologie, 15, pp. 1-83. Hermann, Paris.

1939

141. Leçon inaugurale au Collège de France. Presse méd. p. 41.

142. Sur les rapports fonctionnels entre les hormones mâles et femelles. Monde Med. p. 17. 
143. Sur l'analyse quantitative de l'endocrinologie de la gestation chez la Lapine. (with A. Jost.) C. r. Séanc. Soc. Biol. 130, 726.

144. La conservation de l'utérus a-t-elle de l'importance pour le maintien de l'intégrité des fonctions hormonales de la Femme. Bull. Soc. r. belge Gynéc. Obstet. 2.

145. La prégnéninolone assure le maintien de la grossesse chez la Lapine. (with A. Jost.) C. r. Séanc. Soc. Biol. 130, 1162.

146. Sur l'action androgène de la prégnéninolone. (with A. Jost.) C. r. Séanc. Soc. Biol. 130, 1515.

147. Concentration des produits gonadotropes urinaires par la méthode des mousses. (with A. Dognon.) C. r. hebd. Séanc. Acad. Sci., Paris, 209, 242.

\section{9-1940}

148. Structure et histophysiologie de l'appareil génital femelle. Encyclopédie Médico-Chirurgicale, 3rd edn. 1939-1940.

149. La désoxycorticostérone est capable de maintenir la grossesse ou de provoquer l'avortement. Annls Endocr. 1, (5).

\section{1}

150. Antagonisme et synergie des hormones ovariennes. Bull. Acad. Méd. 124, 39.

151. Action synergique des hormones ovariennes sur la symphyse pubienne des Cobayes. Annls Endocr. $2,124$.

152. Evolution de la grossesse extra-utérine chez la Lapine castrée, C. r. Séanc. Soc. Biol. 135, 820.

153. Action de la progestérone chez la souris mâle castrée. (with H. Bennetz.) C. r. Séanc. Soc. Biol. 135, 825.

154. Privation hormonale et menstruation. (with M.P. Helmer-Dupic.) Annls Endocr. 2, 63.

155. Les conceptions actuelles sur la menstruation. Paris méd. 31-32, 63.

156. Concentration de la gonadotrophine urinaire de l'homme castré par le procédé des mousses. (with G. Poumeau-Delille \& F. de Rudder.) C. r. Séanc. Soc. Biol. 135, 1034.

157. Sur le mécanisme d'ouverture de la symphyse pubienne en fin de grossesse chez le Cobaye. Bull. Acad. Med. 125, 230.

158. Antagonisme hormonal entre prégnèninolone et folliculine. (with G. Poumeau-Delille.) C. r. Séanc. Soc. Biol. 135, 1315.

159. Sur les actions respectives de la progestérone et de la prégnèninolone chez le mâle castré. (with $\mathbf{H}$. Bennetz.) Annls Endocr. 2, 253.

1942

160. Le déciduome provoqué et l'ouverture de la symphyse pubienne chez le Cobaye. (with G. Poumeau-Delille.) C. r. Séanc. Soc. Biol. 136, 138.

161. L'action kératinisante de la désoxycorticostérone sur le vagin et les différentes propriétés des trois substances hormonales progestatives. C. r. Séanc. Soc. Biol. 136, 196.

162. Action de la désoxycorticostérone sur la surrénale. (with G. Poumeau-Delille.) C. r. Séanc. Soc. Biol. 136, 261.

163. Sur l'action androgène de l'acétate de désoxycorticostérone. (with $\mathrm{H}$. Bennetz.) Annls Endocr. 3, 118.

164. Action de quelques stéroïdes sur le tractus génital mâle. (with G. Poumeau-Delille.) C. r. Séanc. Soc. Biol. 136, 360.

165. Propriétés androgènes de la prégnèninolone. Masculinisation d'embryons de lapin par la prégnèninolone injectée à la mère en gestation. (with A. Jost.) Bull. Soc. zool. Fr. 67, 96.

166. Intersexualité foetale provoquée par la prégnèninolone au cours de la grossesse. (with A. Jost.) C. r. Séanc. Soc. Biol. 136, 395.

167. Remarques à propos de différentes actions exercées par certains stéroïdes. Annls Endocr. 3, 181.

168. Notions sur l'endocrinologie de la grossesse. Paris Med. 31-32, 245.

\section{3}

169. Remarques sur l'action androgène de quelques stéroïdes. C. r. Séanc. Soc. Biol. 137, 53.

170. Sur l'action vaginale de l'acétate de désoxycorticostérone et de quelques autres stéroïdes. $C$. $r$. Séanc. Soc. Biol. 137, 94.

171. La prégnèninolone ou éthinyltestostérone. Revue fr. Gynéc. p. 201.

172. Action de la déhydroandrostérone sur le vagin. Annls Endocr. 4, 248.

173. Antagonisme hormonal par action directe et localisée sur une partie du récepteur. (with G. Poumeau-Delille.) C. r. Séanc. Soc. Biol. 137, 361.

174. Action de la progestérone purifiée chez le chat mâle. C. r. Séanc. Soc. Biol. 137, 587. 
175. Sur la polyvalence hormonale de la prégnèninolone. (with A. Jost \& G. Poumeau-Delille.) C. r. Séanc. Soc. Biol. 137, 683.

176. Action de la progestérone purifie sur la gestation. (with H. Bennetz.) C. r. Séanc. Soc. Biol. 137, 710.

177. Physiologie Ovarienne. In Sujets médicaux d'actualité. Ed. Pasteur Vallery-Radot. Doin, Paris.

\section{4}

178. Réaction du vagin et antagonisme hormonal, (with G. Poumeau-Delille.) C. r. Séanc. Soc. Biol. 138, 81.

179. Sur l'obtention de la thyroxine marquée par le radioiode et son comportement dans l'organisme. (with F. Joliot, A. Horeau \& P. Süe.) C. r. hebd. Séanc. Acad. Sci., Paris, 218, 769.

180. Action du propionate de testostérone sur la gestation chez la Lapine. (with A. Jost.) C. r. Seanc. Soc. Biol. 138, 285.

181. Sur la préparation d'une hormone contenant des atomes radioactifs. (with F. Joliot, A. Horeau \& P. Süe.) C. r. Séanc. Soc. Biol. 138, 325.

182. Sur les propriétès de l'androstandiènolone. (with L. Velluz \& J. J. Alloiteau.) C. r. Séanc. Soc. Biol. 138, 496.

183. Sur l'éthylfolliculine. (with L. Velluz \& J. J. Alloiteau.) Annls Endocr. 5, 202.

\section{5}

184. Sur les éthers-oxydes éthyliques de l'oestrone et de l'oestradiol. (with L. Velluz, J. J. Alloiteau \& G. Rousseau.) C. r. Séanc. Soc. Biol. 139, 128.

185. Action de l'aminothiazol sur la synthèse des substances thyroïdiennes. (with F. Joliot, D. Bovet, A. Horeau, G. Poumeau-Delille \& P. Süe.) C. r. Séanc. Soc. Biol, 139, 278.

186. Thyroïde, aminothiazol et iode radioactif. (with F. Joliot, D. Bovet, A. Horeau, G. PoumeauDelille \& P. Sue.) Soc. méd. Hopitaux, séance 18 mai, p. 213.

187. Nouvelles remarques sur les rapports fonctionnels entre folliculine et progestérone. (with G. Poumeau-Delille.) Annls Endocr.

188. Structure moléculaire et activité oestrogène. Etude de structures apparentées à celles du triphényléthylène. (with J. Jacques \& G. Poumeau-Delille.) Bull. Soc. Chimie biol. 32, 373.

189. Présentation d'un jeune déporté français castré au camp d'Auschwitz, en 1943. (with G. Poumeau-Delille.) Bull. Acad. Med. 129, 414.

190. Influence de la thyroxine sur la pénétration de l'iode radioactif dans la thyroïde. (with F. Joliot, P. Süe \& A. Horeau.) C. r. Séanc. Soc. Biol. 139, 657.

191. Radioactivité artificielle et endocrinologie. Schweiz. med. Wschr. 75, 846.

192. Sur la polyvalence des stéroïdes. C. r. Séanc. Soc. Biol. 139, 971.

193. Endocrinologie de la Gestation. Masson, Paris, $400 \mathrm{pp}$.

194. Les corrélations fonctionnelles entre substances stéroïdes. In Exposés annuelés de Biochimie médicale, Ser. 6. Masson, Paris.

195. Sur quelques travaux Américains récents en endocrinologie. Bull. Acad. Méd. 130, 288.

196. Sur l'activité oestrogène d'un acide-phénol apparenté aux hormones sexuelles femelles. (with $A$. Horeau.) C. r. Séanc. Soc. Biol. 140, 332.

197. A propos d'un exposé de M. Moricard. C. r. Séanc. Soc. Biol. 140, 379.

\section{7}

198. Oestrogens and gonadotrophic extracts. F. Endocr. 5, xviii.

199. Sur un nouvel oestrogène artificiel de grande activité. (with A. Horeau \& J. Jacques.) C. r. Séanc. Soc. Biol. 141, 159.

200. L'acide allénolique et ses dérivés. (with A. Horeau \& J. Jacques.) C. r. hebd. Séanc. Acad. Sci., Paris, 224, 1401.

201. Relations fonctionnelles entre substances gonadotropes et oestrogènes. Acta anat. 4, 94.

202. Sur un oestrogène artificiel nouveau. (Review).

203. Discussion du rapport de M. Moricard sur l'ovulation. In 10th Congr. Fr. Gynéc. pt 2, 18. L'expansion scientifique française, Paris.

204. Action de l'acide diméthyl-éthyl-allénolique chez la femelle de cobaye qui allaite. (with A. Horeau \& J. Jacques.) C. r. Séanc. Soc. Biol. 141, 747.

205. L'acide diméthyl-éthyl-allénolique. Fournées Therapeutiques, Paris, p. 21.

206. Quelques aperçus sur la question des hormones. Lecture à la Séance publique des 5 Académies. 207. La relaxine existe-t-elle? (with M. Marois.) C. r. Séanc. Soc. Biol. 141, 1202.

\section{8}

208. Influence des récepteurs dans la comparaison des activités de divers oestrogènes. (with A. Horeau \& J. Jacques.) C. r. Séanc. Soc. Biol. 142, 146. 
209. Le problème de la relaxine. Volume Jubilaire G. Cotte.

210. Sur un oestrogène radioactif. (with A. Horeau, J. Jacques \& P. Süe.) C. r. hebd. Séanc. Acad. Sci., Paris, 227, 8.

211. Sur un oestrogène artificiel nouveau. Congr. int. Physial. Path. Reprod. anim., Milan.

212. Discours sur A. Lacroix. Séance des Prix de l'Académie des Sciences, décembre 1948.

\section{9}

213. Conférence "Hormones Artificielles". Palais de la Découverte.

214. L'hyaluronidase. Orientn. méd. 2, 10.

215a. Sur la préparation et le métabolisme d'iodocaséines radioactives d'inégale activité biologique. (with J. Roche, G. H. Deltour, M. Marois, R. Michel \& F. Morel.) C. r. Seanc. Soc. Biol. 143, 597.

215b. Sur l'excrétion mammaire d'iode radioactif après administration d'iodures ou d'iodocaséine marquée. (with J. Roche, G. H. Deltour, M. Marois, R. Michel \& F. Morel.) C. r. Seanc. Soc. Biol. 143, 599.

216. Etude quantitative de la pénétration de la radiothyroxine dans les cellules hypophysaires. (with A. Horeau, M. Marois \& F. Morel.) C. r. Séanc. Soc. Biol. 143, 935.

217. Hormones radioactives et récepteurs. (with A. Horeau, J. Jacques, M. Marois, F. Morel \& $\mathbf{P}$. Süe.) C. r. hebd. Séanc. Acad. Sci., Paris, 229, 275.

218. Sur la structure des lactones produites par oxydation de l'oestrone. (with A. Horeau \& J. Jacques.) C. r. hebd. Séanc. Acad. Sci., Paris, 229, 321.

1950

219. Interactions between oestrogen and progesterone. Vitams Horm. 8, 179.

220. Effet de la castration sur la gravidité du cobaye. In Book in Honour of Professor Ludwig Fraenkel, p. 103. Montevideo.

221. Nouvelles remarques concernant l'effet de l'ovariectomie sur la gestation. (with R. Colonge.) C. r. hebd. Seanc. Acad. Sci., Paris, 230, 1438.

222. Sur quelques anomalies de la gestation obtenues par mécanisme hormonal. Trans. Int. Ë 4th Am. Congr. Obstet. Gynec., New York. (Am. F. Obstet. Gynec. 61A-1951.)

223. Allenolic acid, a new estrogen. National Research Council, Committee on Human Reproduction, New York.

224. Notice sur la vie et les travaux de René Maire. Séance des Prix de l'Académie des Sciences, décembre 1950.

\section{1}

225. Interrelations entre les hormones génitales femelles. Revue méd. Suisse romande, 1.

226. Sur la penétration de la thyroxine dans le lobe postérieur de l'hypophyse. (with A. Horeau, M. Marois \& F. Morel.) C. r. hebd. Séanc. Acad. Sci., Paris, 231, 776.

227. Contribution à l'endocrinologie de la thyroïde. Acta endocr., Copenh. 7, 54.

228. Cortisone et gestation chez la lapine. (with R. Colonge.) C. r. hebd. Séanc. Acad. Sci., Paris, 232, 1164.

229. Oestrogènes artificiels: nouveau dérivé très actif dans la série allénolique. (with $\mathrm{A}$ Horeau \& $\mathrm{J}$. Jacques.) C. r. hebd. Séanc. Acad. Sci., Paris, 232, 2382.

230. Action de la cortisone administrée à la mère sur la surrénale du foetus de rat. (with $R$. Colonge \& M. Baclesse.) C. r. hebd. Séanc. Acad. Sci., Paris, 233, 333.

231. A propos des problèmes de l'hormonologie sexuelle. (with M. Marois.) Schweiz. med. Wschr. 41, 989.

232. Sur une équilénine synthétique dépourvue de noyau C. (with A. Horeau \& J. Jaques.) C. r. hebd. Séanc. Acad. Sci., Paris, 233, 1542.

1952

233. Relations entre la testostérone et la cortisone dans leurs effets sur certains organes récepteurs. (with M. Marois.) C. r. hebd. Séanc. Acad. Sci., Paris, 234, 271.

234. Contribution nouvelle au problème des relations entre la structure chimique et l'activité oestrogène. (with J. Jacques.) C. r. Séanc. Soc. Biol. 146, 217.

235. Sur quelques anomalies de la gestation obtenues par mécanisme hormonal. Archs Anat. Histol. Embryol. 34, 145.

236. Nouvelles recherches sur les relations de la désoxycorticostérone et de la progestérone. (with $\mathbf{R}$. Colonge.) C. r. Séanc. Soc. Biol. 146, 979.

237. Cortico-surrénale et gestation. (Symposium de Québec.)

238. Notice sur la vie et les travaux de Lucien Cuénot. Séance des Prix de l'Académie des Sciences, décembre 1952.

239. Applications de la mécanique ondulatoire. Témoignage d'un Biologiste. In Louis de Broglie, physicien et penseur. Albin Michel, 1952.

C 
240. Structure chimique et activité oestrogène dans le groupe des isomères de l'acide $\alpha$ - $\alpha$-diméthyl- $\beta$ éthylallenolique. (with J. Jacques.) C. r. hebd. Séanc. Acad. Sci., Paris, 236, 1716.

1953

241. Rapports de la cortico-surrénale et de la sexualité. (with M. Baclesse \& M. Marois.) Ass. Physiologistes. 45, 327.

242. Introduction à l'emploi des radioisotopes. Sem. Hôp. Paris, 28, 1397.

243. Notice nécrologique sur M. J. Jolly. Archs Anat. microsc. 42, 1.

244. Rapports fonctionnels entre l'acide diméthyl-éthyl-allénolique et la progestérone Nouvelles recherches. (with R. Colonge \& L. Chédid.) C. r. Séanc. Soc. Biol. 147, 944.

245. La série allénolique des oestrogènes artificiels. Livre Jubilaire d'A. Lipschutz. Acta physiol. latinoam. 3,71 .

246. Effets comparés de l'acide diméthyl-éthyl-allénolique et de l'oestradiol sur la gestation chez la souris. (with R. G. Freire.) C. r. hebd. Séanc. Acad. Sci., Paris, 237, 949.

\section{4}

247. Quelques faits enregistrés dans mon laboratoire sur la cortico-surrénale. In fubilee Book for Celestino de Costa. Gazeta medica portuguesa, p. 178.

248. Cortisone et sexualité femelle. Annls Endocr. 15, 1.

249. Notions de radio-endocrinologie. Stras. méd. 5, 55.

250. Action de l'hypothermie expérimentale sur la gestation chez le rat. C. r. Séanc. Soc. Biol. 147, 1922.

251. Mesure de l'activité physiologique de la glande thyroïde. (with F. Morel, R. Colonge \& S. André.) C. r. hebd. Séanc. Acad. Sci., Paris, 238, 423.

252. Hommage au Professeur Aschheim. Annls Endocr. 15, 243.

253. Une méthode indirecte permettant de mesurer la fonction thyroïdienne chez le rat. (with $F$. Morel \& R. Colonge.) Annls Endocr. 15, 751.

254. Retard de la nidation et du développement foetal chez la rate en hypothermie. (with M. Marois.) Annls Endocr. 15, 738.

255. Modèles ouverts des acides bis-déhydrodoisynoliques. (with A. Horeau, J. Jacques \& R. Gay.) C. r. hebd. Séanc. Acad. Sci., Paris, 238, 2476.

256. Sur les propriétés biologiques des allénolates de désoxycorticostérone. (with A. Horeau.) C. $r$. hebd. Séanc. Acad. Sci., Paris, 239, 14.

257. Les allénolates de désoxycorticostérone. Livre jubilaire de Westman. Acta endocr., Copenh. 17, 54.

258. Appréciation de l'activité thyroidienne. Livre jubilaire de Bounoure.

259. Action des hormones thyroïdiennes sur la fixation des iodures marqués par le corps thyroïde du rat normal ou hypophysectomisé. (with J. Roche, O. Michel, R. Michel \& R. Colonge.) C. r. Séanc. Soc. Biol. 148, 1144.

260. Notice sur Eugène Bataillon. Séance des Prix de l'Académie des Sciences, décembre 1954.

\section{5}

261. Recherches de mon laboratoire sur la thyroïde. F. Med. Bordeaux, 132, 97.

262. Action des hormones thyroïdiennes et de la thyréostimuline sur la fixation des iodures par le corps thyroïde du rat hypophysectomise. (with J. Roche, O. Michel, R. Michel \& R. Colonge.) C. $r$. Séanc. Soc. Biol. 149, 307.

263. Evaluation de Ia fonction thyroïdienne par l'iode radioactif en clinique humaine: comparaison des résultats fournis par deux tests simples. (with M. Tubiana \& F. Morel.) Bull. Acad. natn. Med. $139,177$.

264. Hormones thyroïdiennes et fixation des iodures par le corps thyroïde. (with J, Roche, O. Michel, R. Michel \& R. Golonge.) Bull. Soc. Chim. biol. 37, 439.

265. Iode radioactif et fonction thyroïdienne. Revue de l'Umfia, 167, 95.

266. L'équilibre hormonal au cours de la gestation. (with M. Baclesse.) Rapport $3^{\circ}$ Réunion Endocrinologistes de langue française, Bruxelles, pp. 1-16, Masson et Cie, Paris.

267. L'oestrogène interrompt la gestation chez certaines espèces par antagonisme hormonal. (with E. Sakiz.) C. r. hebd. Seanc. Acad. Sci., Paris, 241, 1364.

\section{6}

268. Sur la distribution de la cortisone marquée chez le lapin. (with L. Zizine.) C. r. hebd. Séanc. Acad. Sci., Paris, 242, 315.

269. Structure moléculaire et activité œstrogène: acides allénoliques diversement disubstitués en $\alpha$. (with A. Horeau \& R. Gay.) C. r. hebd. Séanc. Acad. Sci., Paris, 242, 429. 
270. Contribution to the regulation of thyroid activity. In Regulation and Mode of Action of Thyroid Hormones, pp. 21-30. Ciba Fdn Colloq. Endocr. 10. Eds. G. E. W. Wolstenholme and E. C. P. Millar. Churchill, London.

271. Action de diverses hormones thyroïdiennes et de l'acide $3: 5: 3^{\prime}$ triiodothyro-acétique sur la fixation des iodures dans le corps thyroïde. (with J. Roche, O. Michel, R. Michel \& A. Colonge.) C. r. hebd. Séanc. Acad. Sci., Paris, 243, 5.

272. Sur la pénétration des hormones thyrö̈diennes et de l'acide $3: 5: 3^{\prime}$ triiodothyroacétique dans l'hypophyse de lapin. (with J. Roche, O. Michel, R. Michel \& A. Colonge.) C. r. Séanc. Soc. Biol. $150,1125$.

273. Remarque sur la greffe d'hypophyse. Jubilé Pol Gérard. Archs Biol. Paris, 67, 461.

274. Contribution à l'étude de l'action de la posthypophyse sur l'activité thyroïdienne du rat. (with L. Zizine.) Congr. int. Physiol., Bruxelles.

275. Action des oestrogènes, des androgènes et des corticostéroides sur l'activité thyroïdienne. (with $F$. Morel, L. Zizine \& A. Psychoyos.) C. r. hebd. Séanc. Acad. Sci., Paris, 243, 217.

276. Sur la régulation hormonale de la fixation thyroidienne des iodures et sur la pénétration des hormones thyroïdiennes dans l'hypophyse. (with J. Roche, O. Michel, R. Michel \& A. Colonge.) Bull. Soc. Chimie biol. 38, 1245.

277. Action de la thyroxine sur la thyroïde d'animaux porteurs de lésions hypothalamiques. (with $F$. Morel \& A. Colonge.) C. r. hebd. Séanc. Acad. Sci., Paris, 243, 333.

278. Substances antihistaminiques et fonction du corps jaune de l'ovaire. (with L. Chédid.) C. r. hebd. Séanc. Acad. Sci., Paris, 243, 1001.

279. Nouvelle contribution à l'etude des rapports entre la posthypophyse et la thyroide. (with L. Zizine.) C. r. hebd. Séanc. Acad. Sci., Paris, 243, 1577.

280. Notice sur Winogradsky. Séance des Prix de l'Académie des Sciences, décembre, 1956.

\section{7}

281. Remarques sur le fonctionnement de la thyroïde foetale chez le lapin. (with L. Zizine.) C. r. hebd. Séanc. Acad. Sci., Paris, 245, 258.

282. Résultats de greffes hypophysaires chez le rat. (with A. Colonge.) C. r. hebd. Séanc. Acad. Sci., Paris, $245,388$.

283. Sur la pénétration de la D-thyroxine et de la $3: 5: 3^{\prime}$ triiodo-D-thyronine dans l'hypophyse, et sur la fixation thyroïdienne des iodures sous leur influence. (with J. Roche, O. Michel, R. Michel \& A. Colonge.) C. r. hebd. Séanc. Acad. Sci., Paris, 245, 1356.

284. Sur la dissociation des diverses stimulations d'origine préhypophysaire. Acta physiol. pharmac. néerl. 6,641 .

285. Greffes et reliquats hypophysaires. Revue stisse Zool. 64, 589.

286. Le Collège de France. Nouva Antol. 1882, 207.

\section{8}

288. Nouvelle contribution à l'étude du fonctionnement de la thyroïde foetale chez le lapin. (with L. Zizine.) C. r. hebd. Séanc. Acad. Sci., Paris, 246, 2976.

289. Notice sur René Leriche. Séance des Prix de l'Academie des Sciences, décembre 1958.

290. Ouverture du Symposium et note sur la nature des besoins en animaux pour les recherches endocrinologiques. In ICLA Symposium, 1958, Les animaux des recherches biologiques, (sous les auspices de l'UNESCO) 6-9 octobre 1958.

\section{9}

291. Recherches de mon laboratoire sur la thyroide. Boln. Acad. nac. Med. B. Aires, novembre, 232.

292. L'aldostérone possède-t-elle des propriétés progestatives. (with A. Colonge \& F. Morel.) Archs Anat. microsc. Morph. exp. 48 bis, 57.

293. Nouveaux résultats de greffes hypophysaires. (with A. Colonge.) C. r. hebd. Séanc. Acad. Sci., Paris, $249,2449$.

1960

294. Isolement des hormones hypophysaires: purification de l'hormone folliculo-stimulante de mouton. (with M. Jutisz, C. Hermier \& A. Colonge.) C. r. hebd. Séanc. Acad. Sci., Paris, 250, 431.

295. Etude histologique de greffes hypophysaires manifestant une activité gonadotrope. (with $\mathbf{M}$. Herlant \& A. Colonge.) C. r. hebd. Séanc. Acad. Sci., Paris, 250, 431.

296. Recherches sur la réaction déciduale expérimentale. (with A. Psychoyos). C. r. hebd. Séanc. Acad. Sci., Paris, 250, 2486.

297. Etude histologique de greffes hypophysaires manifestant une activité gonadotrope. (with $\mathbf{M}$. Herlant \& A. Colonge.) C. r. hebd. Séanc. Acad. Sci., Paris, 250, 1770. 
298. Editorial. Revue endocr. clin. 1, 2.

299. Action de l'hormone mélanophorotrope purifiée $(\alpha-M S H)$ sur la fonction thyroïdienne chez le lapin. (with G. Cehovic.) C. r. hebd. Séanc. Acad. Sci., Paris, 251, 832.

300. Sur certaines lésions produites par les aliments qui dérivent des graines de lin. (with A. Colonge.) C. r. hebd. Séanc. Acad. Sci., Paris, 251, 2842.

\section{1}

301. Nécessité et danger des hormones. Revue lyonn. Méd. 10, 239.

302. Étude du mécanisme de l'inhibition des cellules gonadotropes de l'hypophyse par le testicule chez le rat. (with A. Colonge, M. Herlant \& J.L. Pasteels.) C. r. hebd. Séanc. Acad. Sci., Paris, 252, 487.

303. Nouvelles recherches histologiques sur des greffes hypophysaires après une survie prolongée chez le rat hypophysectomisé. (with A. Golonge, M. Herlant \& J. L. Pasteels.) C. r. hebd. Séanc. Acad. Sci., Paris, 252, 645.

304. Etudes d'endocrinologie. Séminaires sur les gonadotrophines et la fonction corticotrope, Vol. I.Hermann, Paris.

305. Réponse corticosurrénalienne à l'hormone hypophysaire corticotrope chez le rat en fonction du sexe. Etudes in vivo. (with R. Guillemin, A. Colonge \& E. Sakiz.) C. r. hebd. Séanc. Acad. Sci., Paris, 252, 3520.

306. Isolement des hormones hypophysaires: obtention de l'hormone folliculo-stimulante de mouton à l'etat hautement purifié. C. r. hebd. Seanc. Acad. Sci., Paris, 252, 3922.

307. Présence dans un extrait d'hypothalamus d'une substance qui stimule la sécrétion de l'hormone antéhypophysaire de lutéinisation (LH). (with R. Guillemin, M. Jutisz, E. Sakiz \& P. Aschheim.) C. r. hebd. Séanc. Acad. Sci., Paris, 253, 922.

\section{2}

308. Sur des lésions testiculaires observées chez le rat blanc. Archs Anat. Histol. Embryol. 54, Suppl., p. 67.

309. Nouvelle méthode d'extraction de la gonadotropine urinaire humaine de ménopause. (with $\mathbf{M}$. Jutisz, M. Théoleyre \& A. Colonge.) Bull. Soc. Chim. biol. 44, 83.

310. La parthénogenèse expérimentale chez les vertébrés et l'oeuvre d'Eugène Bataillon. Padova Universita Degli Studi, Accademia Patavina Di Scienze Lettere ed Arti.

311. La vie et l'oeuvre de Pol Bouin. Séance des Prix de l'Académie des Sciences, décembre 1962.

312. Sur des anomalies thyroïdiennes et testiculaires observées dans un élevage de rats blancs. In Perspectives in Biology, p. 222. Elsevier, Amsterdam.

313. Un entretien avec le Docteur Robert Courrier. Medica, 22, 2.

314. Robert Courrier. Triangle, 5, 306.

1963

315. Isolement des hormones hypophysaires: purification de l'hormone folliculo-stimulante de mouton par filtration sur gel séphadex. (with M. Jutisz, C. Hermier \& A. Colonge.) C. r. hebd. Séanc. Acad. Sci., Paris, 256, 3922.

316. Note sur la greffe d'hypophyse antérieure. Koninkl. Nederl. Acad. Van Wetens-Chappen, Amsterdam, Ser. C, 65,366 .

317. Présence dans un extrait salin d'hypothalamus d'une activité folliculo-stimulante de type FSH. (with A. Colonge, E. Sakiz, R. Guillemin \& M. Jutisz.) C. r. hebd. Séanc. Acad. Sci., Paris, 257, 1206.

318. A propos de la présence d'activités hormonales de type antéhypophysaire dans les extraits d'hypothalamus. (with M. Jutisz \& A. Colonge.) C. r. hebd. Séanc. Acad. Sci., Paris, 257, 3774.

319. Réflexions d'un endocrinologiste. Revue gén. Sci. pur. appl. 70, 195.

320. Pol Bouin (1870-1962). Annls Endocr. 24, 533.

321. Purification de la gonadotropine urinaire humaine de ménopause (HMG) par chromatographie sur amberlite IRG-50 et diéthylamino-éthyl-cellulose. (with M. Jutisz, M. Théoleyre \& A. Colonge.) C. r. hebd. Seanc. Acad. Sci., Paris, 257, 991.

1964

322. Le corps jaune. Annls Endocr. 25, 138.

323. La traversée placentaire des stéroìdes. Leur action sur le foetus. La traversée mammaire. Bull. schweiz. Akad. med. Wiss. 20, 451.

324. Sur la purification de l'hormone folliculo-stimulante hypophysaire (FSH) de mouton et de la gonadotropine urinaire humaine de ménopause (H.M.G.). Acta endocr., Copenh. Suppl. 90, 29.

325. Les Sciences de la Vie. Introduction. Histoire générale des Sciences (R. Taton). La Science contemporaine. Le XX ${ }^{\mathbf{e}}$ siècle. Presses universitaires de France. 2, 605. 
326. Action de l'hormone hypophysaire folliculo-stimulante de mouton chez le rat mâle hypophysectomisé. (with A. Colonge, G. Hermier \& M. Jutisz.) C. r. hebd. Séanc. Acad. Sci., Paris, 259, 643.

327. Séparation des activités folliculo-stimulante et lutéinisante dans une préparation de gonadotropine urinaire humaine de ménopause. (with M. Jutisz, M. Théoleyre \& A. Colonge.) C. $r$. hebd. Séanc. Acad. Sci., Paris, 259, 1195.

328. Activité endocrinienne d'une tumeur de la glande interstitielle du testicule transplantable chez le rat. (with M. R. Rivière \& A. Colonge.) C. r. hebd. Séanc. Acad. Sci., Paris, 259, 1347.

329. Purification et propriétés physico-chimiques et biologiques de I'hormone folliculo-stimulante (F.S.H.) de mouton. (with M. Jutisz, C. Hermier \& A. Colonge.) Abstracts, 6th Int. Congr. Biochem., New York.

330. Effet de l'hypophysectomie pratiquée après la prise de la greffe, sur une tumeur transplantable de la glande interstitielle du testicule chez le rat. (with M. R. Rivière \& A. Colonge.) C. r. hebd. Séanc. Acad. Sci., Paris, 259, 3607.

331. Notice sur la vie et l'oeuvre de G. Bertrand. Séance des Prix de l'Académie des Sciences, décembre 1964.

332. Hormones et gestation. Reflexions d'un endocrinologiste. Rc. Accad. naz. 15, 9.

\section{5}

333. Purification et propriétés physico-chimiques et biologiques de l'hormone hypophysaire folliculostimulante de mouton. (with M. Jutisz, G. Hermier \& A. Colonge.) Séance spéciale de la Société d'Endocrinologie du 4 février.

334. Sur l'origine de l'hormone mâle du testicule. Discussions anciennes et apports nouveaux. Année biol. 4th Ser., 4, 71 .

335. Remarque à propos de la note de M. R. Rivière, A. Priore, F. Berlureau, M. Fournier et M. Guérin. C. r. hebd. Séanc. Acad. Sci, Paris, 260, 2639.

336. Action sur l'hypophyse d'une tumeur transplantable des cellules interstitielles du testicule. (with M. Herlant, M. R. Rivière \& A. Colonge.) C. r. hebd. Séanc. Acad. Sci., Paris, 260, 3153.

337. Action de F.S.H. purifiée sur l'ovaire. In Les fonctions endocriniennes de l'ovaire. Paris.

338. Le rôle des académies dans la promotion de la Recherche scientifique. Avenirs, Les carrières de la Recherche scientifique, 160, 161, 162.

339. Préface. In Le C.N.R.S.: 25 ans de Recherche scientifique. La Documentation française.

340. Préface. In Cahier de Notes 1850-1860. Edition intégrale du "Cahier Rouge" de Claude Bernard, présenté et commenté par M.D. Grmek. Gallimard, Paris.

341. Préface. In Association Claude Bernard des Hôpitaux de Paris. Anniversaire.

342. Allocution Présidentielle. C. r. Séanc. Soc. Biol. 160, 6.

343. Purification et propriétés physico-chimiques et biologiques de l'hormone folliculo-stimulante de mouton. (with M. Jutisz, G. Hermier \& A. Colonge.) Annls Endocr. 26, 670.

\section{6}

344. La fonction endocrinienne du testicule. Mém. Acad. r. Med. Belg. 6, 351.

345. Notice sur la vie et les travaux de Paul Portier. Seance des Prix de l'Académie des Sciences, décembre 1966.

346. Action of purified FSH on the ovary. Eur. Rev. Endocr. Suppl. 2, p. 3.

347. Tumeurs interstitielles expérimentales du testicule. Actual. endocr., Journées de la Pitié, p. 11.

\section{7}

348. Réflexions sur l'action des hormones hypophysaires gonadotropes. Actual. endocr., fournées de la Pitie, p. 35.

1968

349. L'ovaire des mammifères. In Traite de Zoologie, Vol. 16, p. 637. Ed. P.-P. Grassé. Masson, Paris.

350. La gestation. In Traité de Zoologie, Vol. 16, p. 965. Ed. P.-P. Grassé. Masson, Paris.

351. Experimental tumor of the interstitial gland of the testis. In Perspectives in Biology and Medicine, 11, 457. University of Chicago Press.

352. Concerning some recent problems in endocrinology. F. Endocr. 42, iii.

353. Notices sur la vie et les travaux de quelques savants. In Vies laborieuses, vies fécondes. Vol. 1, 239 pp. Gauthiers-Villars, Paris.

354. Réflexions sur le corps jaune. La Physiologie française contemporaine. In Vol. jubilaire du Doyen H. Hermann, p. 293.

355. Les dangers des hormones. Bull. Acad. Med. 156, 4.

356. Les principales découvertes de la Médicine française au cours des cinquante dernières années. $150^{\circ}$ anniversaire de l'Académie national de Médecine. Bull. Acad. Méd. 156, Suppl., p. 11. 